Management international

International Management

Gestiòn Internacional

\title{
De la motivation à l'engagement pour le service public : étude comparative entre la France, la Chine et la Corée du Sud From Public Service Motivation to Public Service Commitment: Comparative case study among China, France and South Korea De motivación de servicio público para el compromiso: estudio comparativo entre Francia, China y Corea del Sur
}

Pierre-Charles Pupion, Hae-Ok Pyun, Kwang-Jai Yun, Philippe Dorbaire, David Huron, Jungho Park et Yugang Guo

Volume 21, numéro 2, hiver 2017

Les nouvelles frontières du management public

The new frontiers of Public Management

Las nuevas fronteras de la gestión pública

URI : https://id.erudit.org/iderudit/1052687ar

DOI : https://doi.org/10.7202/1052687ar

Aller au sommaire du numéro

Éditeur(s)

HEC Montréal

Université Paris Dauphine

ISSN

1206-1697 (imprimé)

1918-9222 (numérique)

Découvrir la revue

Citer cet article

Pupion, P.-C., Pyun, H.-O., Yun, K.-J., Dorbaire, P., Huron, D., Park, J. \& Guo, Y. (2017). De la motivation à l'engagement pour le service public : étude comparative entre la France, la Chine et la Corée du Sud. Management international / International Management / Gestiòn Internacional, 21(2), 60-79. https://doi.org/10.7202/1052687ar
Résumé de l'article

Depuis la théorie de la motivation à l'égard du service public (MSP) de Perry et Wise (1990), de nombreux travaux de recherche ont été menés afin d'identifier des dimensions de la MSP et d'élaborer les échelles de mesure. L'objectif de notre étude est de modéliser le processus motivation-engagement à l'égard du service public, à la lumière de la théorie du comportement planifié (Ajzen, 1991) et du management interculturel (Hofstede, 1984, 1997; Hofstede et Hofstede, 2005). Une étude conduite en France, en Corée du Sud et en Chine montre la spécificité de la MSP par pays et son influence sur l'engagement organisationnel.
Tous droits réservés (C) Management international / International Management / Gestión Internacional, 2017
Ce document est protégé par la loi sur le droit d'auteur. L’utilisation des services d'Érudit (y compris la reproduction) est assujettie à sa politique d'utilisation que vous pouvez consulter en ligne.

https://apropos.erudit.org/fr/usagers/politique-dutilisation/ 


\section{De la motivation à l'engagement pour le service public : Etude comparative entre la France, la Chine et la Corée du Sud}

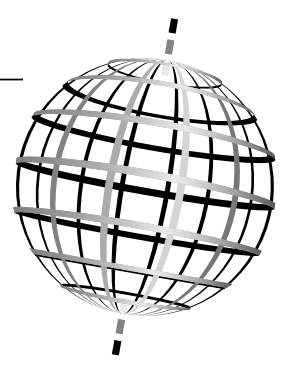

\section{From Public Service Motivation to Public Service Commitment: Comparative case study among China, France and South Korea}

\section{De motivación de servicio público para el compromiso: estudio comparativo entre Francia, China y Corea del Sur}

\author{
PIERRE-CHARLES PUPION \\ Institut de Préparation à \\ l’Administration de Générale, \\ Université de Poitiers, France \\ HAE-OK PYUN \\ Centre de Recherche Juridique de \\ Pothier, Université d'Orléans, France
}

\section{RÉSUMÉ}

Depuis la théorie de la motivation à l'égard du service public (MSP) de Perry et Wise (1990), de nombreux travaux de recherche ont été menés afin d'identifier des dimensions de la MSP et d'élaborer les échelles de mesure. L'objectif de notre étude est de modéliser le processus motivation-engagement à l'égard du service public, à la lumière de la théorie du comportement planifié (Ajzen, 1991) et du management interculturel (Hofstede, 1984, 1997; Hofstede et Hofstede, 2005). Une étude conduite en France, en Corée du Sud et en Chine montre la spécificité de la MSP par pays et son influence sur l'engagement organisationnel.

Mots clés : Motivation à l'égard du service public (MSP), Théorie du Comportement planifié, Culture Nationale, Engagement Organisationnel, Etude comparative

\author{
KWANG-JAI YUN \\ Université d'Yeunginam, Corée du Sud \\ PHILIPPE DORBAIRE \\ Université de Poitiers, France \\ DAVID HURON \\ Université Nice Sophia Antipolis, \\ IAE, GRM France
}

\section{ABSTRACT}

Since the Public Service Motivation theory (Perry and Wise, 1990), numerous research works have been conducted to identify PSM dimensions in order to set up measuring scales. In this study, we aim to model the process of motivation-commitment to public service, in the light of the theory of planned behavior by Ajzen (1991) and Hofstede's cultural management (Hofstede, 1984, 1997; Hofstede et Hofstede, 2005). A study we conducted in France, South Korea and China shows that PSM as well as organizational commitment differ across regions.

Keywords: Public Service Motivation, Planned Behavior, National Culture, Organizational Commitment, Comparative Study

\author{
JUNGHO PARK \\ The Korean Institute of Public \\ Administration, Corée du Sud \\ YUGANG GUO \\ Université de Nanchang, Chine
}

$\mathrm{D}$ epuis la fin des années 1970, les pays occidentaux ont conduit des réformes, inspirées par le New Public Management (NPM). Ces réformes, visant à appliquer les principes de la gestion privée dans les organismes publics ont mis au cœur du changement la performance et ses critères d'efficacité, d'efficience, et d'économie (Hood, 1991; Osborne \& Gaebler, 1993; Dunleavy et Hood, 1994). Ces réformes affectent et même affaiblissent, selon les auteurs du post NPM, la cohérence de l'action publique et la cohésion sociale (Emery \& Giauque, 2012;
Mazouz, Rousseau \& Sponem, 2015). Il s'avère donc nécessaire de rétablir une «éthique commune» et une «culture de cohésion» dans le secteur public (Shergold, 2004) en raison de la méfiance provoquée par la démarche néolibérale qui conduit à une crise des vocations et des recrutements. Mais, quelles sont ces valeurs publiques partagées par les agents publics? Quelle est la source de la motivation pour le service public permettant de développer un réel engagement des agents pour le service public?

\section{RESUMEN}

Basándose en la teoría de la motivación para servicio público (MSP) de Perry y Wise (1990), han sido numerosas las investigaciones llevadas a cabo para identificar las dimensiones de dicha MSP y las medidas correspondientes. Nuestro estudio es una moceso de motivación basándonos en la teoría de la conducta planificada de Ajzen (1991) con el propósito de identificar las palancas que influyen sobre el compromiso en los agentes públicos. Un estudio llevado a cabo en Francia, en Corea del Sur y en China muestra la especificidad de MSP en cada país y su influencia sobre llama formiso organizacional

Palabras clave: La motivación para el servicio público (MSP), teoría de la conducta planificada, cultura nacional, compromiso organizativo, estudio comparativo 
Afin de répondre à ces questions, de nombreuses recherches ont été menées pour identifier les dimensions de la motivation à l'égard du service public (Perry et Wise, 1990; Perry, 1997; Kim, Vandenabeele et al., 2012). Le concept de motivation fait référence aux "forces internes et/ou externes produisant le déclenchement, la direction, l'intensité et la persistance du comportement» (Vallerand et Thill, 1993). Perry et Wise (1990) ont introduit la notion de motivation à l'égard du service public (MSP), qui serait différente de celle des employés du secteur marchand. Elle correspond à une prédisposition individuelle à répondre aux motivations portées en premier lieu, sinon exclusivement, par des institutions et organisations publiques (Perry et Wise, 1990).

Dans notre recherche, nous ne pas cherchons pas à dresser une liste exhaustive des sources de MSP, mais à savoir dans quelle mesure celles-ci peuvent être différentes d'un pays à l'autre et influer sur l'engagement organisationnel. Cette question du lien entre motivation et engagement est fondamentale dans le secteur public où il n'existe pas de forces du marché qui contraignent au changement et où seul l'engagement des agents est garant de sa mise en œuvre (Kim, 2005, Ritz, 2009; Wright et al. 2013). Ainsi, notre question de recherche est «dans quelle mesure la motivation à l'égard du service public (MSP) et les valeurs publiques influent sur l'engagement des agents publics?»

Par engagement, Allen et Meyer (1991) définissent un état psychologique qui caractérise la relation d'un employé avec son organisation et influe sur ses comportements au travail. Par valeurs, sont désignées des normes et références personnelles et/ou collectives qui influencent l'attitude et le comportement de celui ou ceux qui y adhérent. Le système de valeurs est une organisation stable - au long d'un continuum d'importance relative - de croyances portant sur les modes de conduite ou les visées d'un état de vie préférable aux autres.» (Rokeach, 1973, p.5). La motivation est ici appréhendée de façon plus large que ne l'ont abordée les pionniers de la MSP qui mettent l'accent sur la spécificité altruiste de cette motivation des salariés du public. Nous introduisons notamment les facteurs de culture nationale et managériale.

Pour répondre à la question de recherche, nous sollicitons la théorie du comportement planifié (Ajzen, 1991) et ajoutons certains apports des recherches sur les théories de la MSP (Perry, 1996,; Kim, Vandenabeele et al, 2012), du management interculturel (Hofstede, 1984, 1997; Hofstede et Hofstede, 2005), du NPM et de l'engagement. L'étude conduite à partir de trois échantillons d'enseignants en administration et management public en France, en Chine et en Corée du Sud, permet de valider partiellement le modèle par une approche de type PLS et des méthodes d'inférence statistique.

\section{De la motivation à l'égard du service public à l'engagement pour le service public}

\section{DE LA MOTIVATION À L'ÉGARD DU SERVICE PUBLIC...}

Selon Perry et Wise (1990), les comportements de fonctionnaires ne sont pas dictés par les mêmes motivations que les salariés du secteur privé. Pour remplir une mission de service public, il faut que les personnes aient cette motivation à l'égard du service public (MSP), définie comme "la prédisposition d'un individu à répondre à des motivations portées principalement ou uniquement par des institutions ou organisations publiques." (Perry et Wise 1990, p.368). Rainey et Steinbauer (1999, p. 23) la définissent comme "une motivation altruiste générale de servir les intérêts d'une communauté de personnes, un état, une nation, ou le genre humain", tandis que Vandenabeele (2007, p. 547) la définit comme étant «les croyances, les valeurs et les attitudes qui, allant au-delà de l'intérêt personnel et de l'intérêt de l'organisation, expriment le souci de l'intérêt d'une entité politique plus vaste et motivent les individus à agir en conséquence le cas échéant». Selon Vandenabeele et Van de Walle (2008), cette motivation est spécifique aux salariés du public et diffèrerait selon les pays.

Perry et Wise (1990, p.368) se référant aux travaux de Knote et Wright-Isak (1982), considèrent qu'il y a trois formes de la MSP : rationnelle, normative et affective. Si la dimension rationnelle de la motivation conduit à des actions fondées sur la recherche de la maximisation de l'utilité individuelle, la dimension normative se réfère aux actions correspondant aux efforts visant à contraindre les fonctionnaires à se conformer aux normes sociales, et notamment à celles de l'intérêt public, du devoir civique et de la loyauté envers le gouvernement (Downs, 1967). Quant à sa dimension affective, elle repose sur des éléments déclencheurs de comportements fondés sur les réponses émotionnelles à divers contextes sociaux. La bienveillance, la compassion, le sacrifice de soi en sont une illustration.

Perry (1996) construit une échelle de mesure de la motivation, constituée de vingt-quatre éléments associés à quatre facteurs : attirance pour les politiques publiques, engagement pour l'intérêt public, compassion et sacrifice de soi. Cette échelle mesure les dimensions rationnelle, normative et affective de la motivation d'un individu qui cherche un emploi dans le secteur public. En 1997, Perry montre par enquête que la richesse est liée négativement à la MSP et que les expériences au sein de la famille, l'idéologie et le niveau d'éducation constituent des facteurs déterminants pour le développement ultérieur de la MSP.

Les recherches examinant les effets de la MSP sur l'engagement et le comportement des fonctionnaires, donnent des résultats peu convergents. Cette motivation ne semble pas être liée à l'engagement (Edey Gamassou, 2014) qui dépendrait plutôt de l'âge, du niveau de poste occupé, de la perception du soutien de la part du supérieur.

Partant de ces analyses, nous examinons plus en détail la relation entre la MSP, le soutien organisationnel et l'engagement à l'aide de la théorie du «comportement planifié» d'Ajzen (1991).

\section{... À L'ENgAgEMENT DES AgENTS PUBLICS POUR LE SERVICE PUBLIC : LA THÉORIE DU COMPORTEMENT PLANIFIÉ}

En prolongement de la théorie de l'action raisonnée (Ajzen \& Fishbein, 1980; Fishbein et Ajzen, 1975), Ajzen (1991) propose d'intégrer la perception du contrôle sur le comportement pour mieux expliquer et prédire le comportement des individus dans une situation donnée. Selon ce modèle, les attitudes, la perception du contrôle sur le comportement et les pressions sociétales influent sur les intentions de l'individu, qui, à leur tour, façonnent son comportement (voir la figure 1). 


\section{FIGURE 1} Comportement planifié

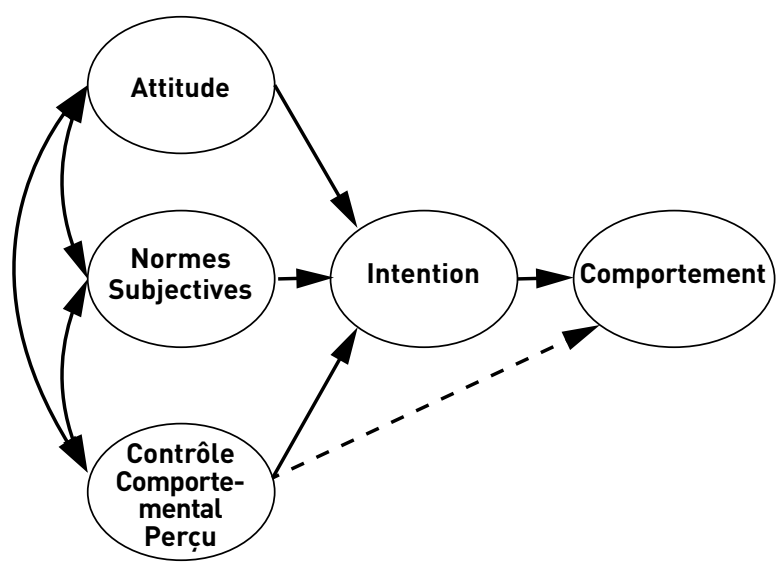

(Source : Ajzen, 1991)

«[...] L'intention d'une personne à exécuter le comportement est d'autant plus forte que l'attitude et la norme subjective sont favorables audit comportement et que la perception du contrôle sur le comportement est grande..., [et] dans certains cas, on peut constater que seules les attitudes ont un impact significatif sur les intentions. Dans d'autres cas, ces attitudes et la perception du contrôle sur le comportement sont suffisantes pour rendre compte des intentions, et dans d'autres cas encore ces trois facteurs prédictifs contribuent de façon indépendante. [...]» (Ajzen, 1991, pp. 188-189).

- Les intentions constituent les antécédents des comportements et sont «des indications de la volonté et des efforts que les gens sont prêt à consentir afin d'exécuter le comportement. [...] Plus est forte l'intention, plus il y a de chance que l'individu y arrive.» (Ajzen, 1991, p.181).

- L'attitude envers un comportement fait référence au degré de jugement positif ou négatif que la personne a de la conduite en question (Ajzen, 1991).

- La norme subjective est la perception de la pression sociale sur l'individu, représentée par les points de vue d'autrui (parents, conjoints, amis, enseignants...) sur le comportement qu'il veut adopter (Ajzen, 1991; Harding et al., 2007).

- La perception du contrôle sur le comportement fait référence à la facilité ou la difficulté perçue concernant la mise en œuvre du comportement. Celle-ci dépendrait de l'autoefficacité ou "confiance qu'une personne a en sa capacité de réaliser les actions nécessaires pour obtenir un résultat désiré» (Bandura, 1977, p.193) et de la perception du soutien organisationnel, ensemble «des croyances [des employés] concernant la capacité de l'organisation à valoriser leurs contributions et à se soucier de leur bien-être » (Eisenberger et al., 1986, p.500). Ces deux dimensions influeraient sur l'intention et le comportement.

Cette théorie permet d'appréhender l'ensemble des effets de la motivation pour le service public en l'incluant dans un processus comportemental complet qui explique pourquoi l'agent public va rester dans son organisation. L'engagement, volonté de rester dans l'organisation, correspond, au sens d'Ajzen, à une intention de rester tandis que le comportement est le fait de rester ou non dans l'organisation. Cette intention s'explique par la perception du contrôle de comportement, par la pression des normes subjectives propres à chaque pays et par la motivation de service public qui correspond à la fois à une attitude et à des normes subjectives.

\section{MODÈLE DE PROCESSUS MOTIVATION-ENGAGEMENT À L'ÉGARD DU SERVICE PUBLIC}

Ce modèle permet de mieux comprendre et identifier le processus motivation-engagement à l'égard du service public. Les différentes dimensions de la MSP, reflets de l'attitude et des normes subjectives, ainsi que la perception du contrôle sur le comportement vont influer directement ou indirectement sur l'engagement qui comprend une dimension affective, calculée et normative (Meyer et Allen, 1991).

Ce processus d'engagement à l'égard du service public est principalement expliqué par (voir la figure 2) :

- Les trois dimensions de la MSP (affective, rationnelle et normative) telles que décrites par Perry et Wise (1990) ou Perry (1996). Les deux premières relèvent d'une attitude ou «d'un jugement positif lié au travail pour le service public» alors que la dimension normative correspond davantage dans la terminologie d'Ajzen aux normes subjectives, reflets de pressions sociales.

- La perception du contrôle sur le comportement qui comporte le sentiment d'auto-efficacité et la perception du soutien organisationnel influe sur l'engagement de fonctionnaires.

- Le niveau de l'emploi occupé et certains facteurs démographiques individuels (Perry, 1997; Edey Gamassou, 2014) qui influent sur l'engagement de l'agent public.

- Les dimensions culturelles qui influent notamment sur la MSP. Nous formulons les hypothèses suivantes :

H 1 : Les dimensions affective, rationnelle et normative de la MSP influent sur l'engagement pour le service public.

Les employés ayant un niveau élevé de motivation pour le service public ont tendance à être plus impliqués (Pandey et Stazyk, 2008; Xiaohua, 2008), car ils sont plus attirés par la politique publique et l'intérêt public et plus disposés à faire le sacrifice de soi (Ritz, 2009; Vandenabeele, 2009; Shrestha et Mishra, 2015).

H 2 : La perception du contrôle sur le comportement influe sur l'engagement.

L'engagement est positivement lié à l'auto-efficacité (Bandura, 1977; Meyer, Stanley, Herscovitch et Topolnytsky, 2002; Sahertian \& Soetjipto, 2011). Les individus sont davantage enclins à participer à des activités et à s'engager dans la mesure où ils se considèrent efficaces pour entreprendre ces activités. De même, l'individu percevant un fort soutien de la part de son organisation, sera par réciprocité prêt à faire davantage d'efforts pour l'organisation (Brunetto et al., 2013) et inversement (Shanock \& Eisenberger, 2006).

H 3 : L'ancienneté et le niveau de l'emploi exercent une action positive sur l'engagement. 


\section{FIGURE 2}

\section{Processus Motivation-Engagement pour le service public}
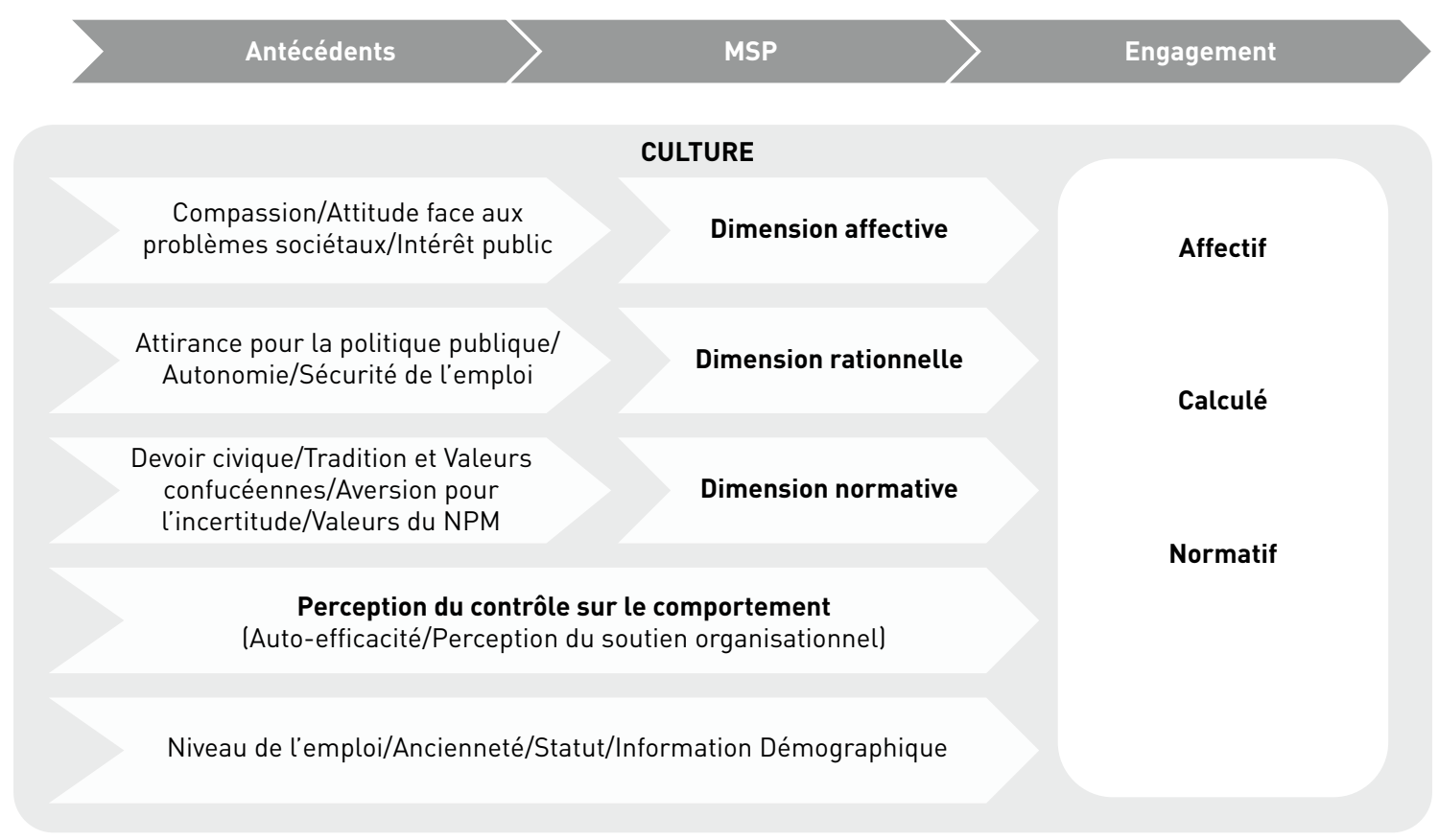

Meyer et Allen (1991) ont considéré que les variables sociodémographiques comme l'âge, le sexe, l'état civil, l'ancienneté et le niveau d'éducation sont susceptibles d'influer sur l'engagement organisationnel. Certaines études prouvent que l'engagement rationnel est influencé par l'âge et l'ancienneté (Tremblay, Guay, Simard, 2000).

\section{CULture Nationale, UNE VARIABLE EXPLiCATIVE DE LA MSP ET DE L'ENGAGEMENT}

Suivant la théorie des dimensions culturelles de Hofstede (1984), nous supposons que la culture nationale a une influence sur les différentes formes motivation de service public et d'engagement. La culture nationale est porteuse d'un ensemble de normes subjectives qui peuvent expliquer des différences en termes de motivation pour le service public (Hondeghem et Vandenabeele, 2005) et notamment sur les dimensions normatives de la MSP.

H 4 : La culture nationale influe sur la motivation et sur l'engagement à l'égard du service public.

House et al. (2004) définissent la culture comme le partage de motifs, de valeurs, de croyances, d'identités et des interprétations qui proviennent d'expériences communes de personnes du même groupe. Ainsi, la culture se trouve évidemment dans les valeurs et les croyances portées par une société donnée (Dunn et Shome, 2009), qui sont holistiques, historiquement déterminées et socialement construites (Detert et al., 2000).

Pour mesurer l'influence de la culture nationale, nous retenons les six dimensions culturelles d'Hofstede (1984, 1997; Hofstede et Hofstede, 2005; Hofstede \& Bond, 1988) et de Fons Trompenaars (1994) : l'Indice de Distance au Pouvoir (PDI), l'Individualisme contre le collectivisme (IDV), la masculinité contre la féminité (MAS), l'Indice d'évitement de l'Incertitude (AUI), le Plaisir contre la Retenue (IND), et l'orientation à court terme contre l'orientation à long terme (LTO) :

- PDI : cet indice évalue dans quelle mesure une personne moins influente accepte l'inégalité dans la répartition du pouvoir et la considère comme normale;

- IDV : cet indice reflète la mesure dans laquelle une société considère ses membres à titre individuel ou en tant que membre du groupe. Dans les sociétés individualistes, les individus sont principalement préoccupés par leurs propres intérêts, alors que dans les sociétés collectivistes, les intérêts du groupe priment;

- MAS : la masculinité caractérise les cultures où les valeurs dominantes sont l'ambition, le pouvoir et la concurrence. Dans la culture féminine, les valeurs dominantes sont la qualité de vie, les relations interpersonnelles, le service, les activités caritatives, la solidarité...

- AUI : cet indice mesure la plus ou moins grande tolérance d'une société vis-à-vis de l'incertitude et de l'ambiguïté. Les sociétés avec un indice AUI d'évitement de l'incertitude élevé sont moins tolérantes face au changement et cherchent à minimiser l'anxiété devant l'inconnu en mettant en place des règles. Les sociétés avec un faible indice d'évitement de l'incertitude sont plus ouvertes au changement et disposent de moins de règles et plus souples.

- IND : cet indice mesure la capacité d'une culture à satisfaire les besoins immédiats et les désirs personnels de ses membres. Les cultures donnant de la valeur à la modération disposent de règles sociales strictes et de normes qui régulent, sinon découragent la satisfaction des pulsions. 
- LTO : à l'origine appelée le «dynamisme confucéen », cette dimension se réfère à la préférence pour les récompenses à long terme ou à court terme. Les cultures axées sur le long terme accordent plus d'importance à l'avenir, les valeurs mises en avant sont celles de la persistance, de l'épargne et de la capacité d'adaptation. A contrario, les sociétés orientées à court terme perçoivent le temps comme circulaire et mettent l'accent sur le passé, le présent, la stabilité, le respect de la tradition et des obligations sociales.

\section{DiMENSION RATIONNELLE DE LA MSP}

Basée sur une évaluation calculatoire des situations et des actions (Knoke et Wright-Isak, 1982), la dimension rationnelle de la MSP est fondée sur le principe de la «maximisation de l'utilité individuelle» (Perry et Wise, 1990, p. 368). Afin d'appréhender cette dimension, nous retenons trois variables : l'attirance pour les politiques publiques, l'autonomie et la sécurité de l'emploi. Nous pensons que ces éléments expliquent la dimension rationnelle de la MSP et influencent l'engagement calculé pour le service public.

\section{Attirance pour la politique publique}

Suivant Perry (1996) et Kim, Vandenabeele et al. (2012), nous considérons que participer à l'élaboration des politiques publiques permettrait aux agents publics de renforcer leur propre pouvoir ou de défendre leur intérêt particulier. Nous pensons également que ce besoin varie selon les pays.

\section{Autonomie (Self-Reliance)}

Suivant Miller, Woehr \& Hudspeth (2002) et Hofstede (1984), nous considérons que cette variable est liée à l'individualisme et évalue dans quelle mesure une personne apprécie d'être indépendante dans son travail quotidien. Les travaux de Goncalo \& Staw (2006) montrent que l'individualisme peut affecter l'engagement organisationnel des employés publics. Tenant compte de l'approche interculturelle, nous pensons que le niveau de l'autonomie varie selon les pays.

\section{Sécurité de l'emploi, ancienneté et niveau de l'emploi}

Reprenant les analyses de Niskanen (1971) pour lequel les fonctionnaires sont mus par la recherche de leur propre intérêt, nous supposons que les agents publics recherchent un statut, du pouvoir, un revenu ainsi que leur propre sécurité d'emploi et des avantages. Selon Baird \& Mainstone (1976) et Edey Gamassou (2014), la sécurité d'emploi, l'ancienneté et le niveau de l'emploi ont un impact sur l'engagement : plus le niveau de l'emploi est élevé et plus l'ancienneté est importante et, plus est forte l'engagement. Nous suggérons que ces variables influent sur le niveau de l'engagement. Dans notre modèle, la recherche de la sécurité d'emploi fait partie de la motivation rationnelle, tandis que l'ancienneté et le niveau d'emploi font partie des informations démographiques.

\section{Dimension AFFECTIVE DE LA MSP}

La dimension affective de la MSP déclenche des comportements pro-sociaux sur la base de réponses émotionnelles aux différentes situations sociales. Afin de la mesurer, nous retenons la compassion, l'attitude face aux problèmes sociétaux et l'intérêt public et suggérons que ces variables influencent l'engagement affectif pour le service public.

\section{Compassion}

Suivant Frederickson et Hart (1985), Perry (1996) suggère que la compassion exprime une réaction émotionnelle à l'humanité. Cette dimension est reprise par Kim, Vandenabeele et al. (2012) dans leur questionnaire sur la mesure de la MSP. Cette compassion, constitutive de la dimension affective de la MSP, influence l'engagement affectif.

\section{Attitude face aux problèmes sociétaux et intérêt public}

Perry (1997) pense que la socialisation est un facteur de la MSP. En outre, Bellah et al. (1986) notent que dans une certaine mesure, l'attitude vis-à-vis des problèmes sociétaux et de l'intérêt public exprime une façon de vivre ensemble. Tenant compte des travaux de Hofstede (1983), Robertson \& Hoffman (2000), et Wu (2006), nous suggérons que la position face à l'individualisme et au collectivisme est un antécédent de la motivation et diffère selon les pays.

\section{DIMENSION NORMATIVE DE LA MSP}

La dimension normative de la motivation de service oblige les employés publics à se conformer aux normes sociales (devoir civique, tradition). Nous incluons également l'attitude à l'égard des valeurs du New Public Management (NPM) dans cette dimension, mais aussi le sacrifice de soi qui a également une dimension normative et affective.

\section{Devoir civique et Sacrifice de soi}

Dans une première approche, nous considérons que le devoir civique peut être un prolongement du sacrifice de soi et que le devoir civique et le sacrifice de soi correspondent à une composante normative de la MSP influencée par les différences culturelles. En effet, si le devoir civique consiste en l'éthique du service public, découlant de la souveraineté de l'État (Buchanan, 1975) et qu'il est considéré comme une composante normative, il a néanmoins une influence sur l'engagement affectif (Julian et al., 2013). Quant au sacrifice de soi, s'il traduit une volonté de servir les autres en allant au-delà des récompenses financières (Perry, 1996), il «a à la fois des composantes affectives et normatives et renvoie à l'altruisme, qui est au cœur du concept de MSP» (Desmarais et Edey Gamassou, 2014).

\section{Tradition/valeurs confucéennes ou orientation à long terme}

Dans les pays asiatiques historiquement touchés par la culture confucéenne, ces valeurs sont habituellement observées sous la forme d'une pression sociale s'exerçant de manière informelle (Wu, 2006). Ces sociétés ont tendance à se concentrer sur le respect de la tradition, le souci de ne pas perdre la face et de remplir ses obligations sociales mais seraient selon Hofstede des sociétés pragmatiques qui seraient capables d'adapter leur tradition au contexte. Dans notre enquête, sont retenues seulement trois valeurs confucéennes (Hofstede \& Bond, 1988; The Chinese Culture Connection, 1987) : le respect de l'ordre social, l'écoute des anciens, et le respect de la tradition. Nous pensons que ces variables diffèrent selon les pays et sont liées positivement à l'engagement normatif. 


\section{Aversion pour l'incertitude (UAI)}

Cette dimension mesure la plus ou moins grande tolérance face à l'incertitude et à l'ambiguïté organisationnelle (Hofstede, 1983). Dans les cultures à indice élevé d'aversion pour l'incertitude, il y a davantage de formalisation écrite, d'utilisation des règles et de procédures pour diminuer l'ambiguïté dans une organisation et rendre les actes des agents prévisibles puisque conformes aux règles.

\section{Valeurs du New Public Management (NPM)}

Tenant compte des travaux de recherche sur les différences culturelles face aux réformes du secteur public et au NPM (Pratchett, 1996; Pollitt \& Bouckaert, 2011), nous avons décidé d'inclure deux normes du NPM paraissant centrales : la satisfaction des besoins des clients-usagers et le bon usage des impôts.

\section{Perception du Contrôle SUR LE Comportement}

\section{Auto-efficacité (Self-Efficacy)}

Selon Bandura (1985), les individus sont des êtres proactifs par l'entremise de l'auto-organisation, de l'autoréflexion et de l'autorégulation. L'auto-efficacité consiste aux «jugements des gens sur leurs capacités à organiser et exécuter des plans d'action nécessaires pour atteindre des objectifs. Ce ne sont pas des compétences, mais une évaluation de ce qu'on peut faire avec ses compétences» (Bandura, 1985, p. 391). Nous pensons que cette variable influe sur l'engagement.

\section{Perception du soutien organisationnel}

Selon Eisenberger, Huntington et al. (1986), l'engagement des employés envers l'organisation est fortement influencé par leur perception du soutien organisationnel vis-à-vis d'eux. La perception du soutien organisationnel n'est pas unidimensionnelle (Commeiras et Fournier, 2001), ce soutien peut provenir de l'organisation, de son supérieur ou de ses collègues (Paillé et al., 2011; Edey Gamassou, 2014). Dans notre étude, tenant compte de l'importance dans l'intention de rester dans l'organisation (Paillé et al., 2011), nous retenons seulement la perception du soutien de la part de l'organisation. La prise en compte des autres formes de soutien, en grande partie fonction du statut des enseignants-chercheurs (professeur et maître de conférences en France), complexifierait trop le modèle.

\section{ENGAgEMENT DES AgENTS POUR LE SERVICE PUBLIC}

Pour Bateman et Strasser (1984, p.95), l'engagement organisationnel «implique la loyauté d'un employé de l'organisation, la volonté de faire des efforts au nom de l'organisation, le degré de congruence avec l'objectif et les valeurs de l'organisation, et le désir de rester membre». Il est selon Meyer et Allen (1991) multidimensionnel et correspond à "un état psychologique qui (a) caractérise la relation de l'employé avec l'organisation, et (b) a des engagements pour la décision de cesser ou de continuer à être membre de l'organisation. » Retenant leur approche, nous distinguons les trois types d'engagement qualifiés d'affectif, de calculé et de normatif et mis en évidence dans le secteur public par Balfour and Weschler (1996).

"L'engagement affectif se réfère à l'attachement émotionnel de l'employéà l'identification et à l'engagement dans l'organisation.»
(Meyer \& Allen, 1991, p. 67). Dans notre étude, nous l'appelons engagement affectif pour le service public car l'attachement est à la fois pour l'organisation dans laquelle il travaille et pour l'administration qu'il sert. Il correspond à une identification à la mission et aux objectifs de l'organisation définis en grande partie par l'administration. L'engagement calculé pour le secteur public, "se réfère à une prise de conscience des coûts associés à quitter l'organisation " soit en l'occurrence l'administration publique, car le coût pour l'agent public est lié principalement au départ de l'administration et non d'une organisation relevant de celle-ci. Il est fortement orienté vers la sécurité. Enfin, "l'engagement normatif reflète le sentiment d'obligation, celui d'être dans le devoir de poursuivre la relation de travail.» (op. cit.), il s'exprime à la fois vis-à-vis de l'organisation mais aussi vis-vis de l'administration publique ainsi que l'atteste l'expression serviteur de l'Etat et l'honneur de servir l'administration.

\section{L'étude empirique sur les sources de l'engagement pour le service public : une comparaison France, Chine et Corée du Sud}

\section{MÉTHODOLOGIE}

Pour valider notre modèle, nous réalisons une étude quantitative sur les trois échantillons en utilisant les approches PLS d'équations structurelles et les méthodes d'inférence statistique non paramétrique. Partant d'études antérieures, nous élaborons un questionnaire avec les échelles de Likert à 5 points (allant de tout à fait en désaccord... à... tout à fait d'accord) et d'autres échelles numériques de notation à 5 points (de pas du tout... à... extrêmement).

Le questionnaire est constitué de 7 blocs de questions (voir l'annexe 1) sur les dimensions de la MSP (affective, rationnelle et normative), de l'engagement pour le service public (affectif, calculé et normatif), de la perception du contrôle sur le comportement (auto-efficacité et perception du soutien organisationnel) et des informations démographiques. Ce questionnaire traduit en français, en chinois et en coréen a été administré en ligne auprès des enseignants-chercheurs en France et envoyé par courriel aux enseignants-chercheurs en management public de la Corée du Sud par l'entremise de la Korean Association for Public Administration. En Chine, nous avons soumis sur place une version papier du questionnaire aux professeurs en management public de quatre universités de la province de Jiangxi car l'administration en ligne du questionnaire a été impossible.

En raison de la longueur du questionnaire, le nombre d'items par échelle multiple a été réduit dès lors que l'information apportée par l'échelle se saturait. La fiabilité et la validité des échelles de mesure ont été vérifiées en appliquant le protocole proposé par Churchill (1979) (voir le tableau 1).

La phase exploratoire comprend d'une part, la formulation d'un ensemble d'items susceptibles de mesurer les concepts étudiés et d'autre part, la purification des échelles afin d'obtenir des mesures fiables. Nous avons lancé une pré-enquête auprès d'un échantillon de 30 enseignants-chercheurs français puis réalisé une analyse factorielle pour vérifier si les échelles étaient unidimensionnelles. Enfin, utilisant le coefficient alpha de Cronbach (1951), nous considérons comme fiables, les échelles dont l'alpha est supérieur à 0,7; et comme acceptables celles dont 
l'alpha est compris entre 0,7 et 0,5. Les échelles dont l'alpha est inférieur à 0,5 sont purifiées en supprimant certains items. La même démarche a été appliquée au niveau des trois échantillons définitifs et prolongée par une analyse confirmatoire qui permet de vérifier la stabilité, la fiabilité et la validité des instruments par l'utilisation de méthodes d'équations structurelles.

\section{Résultats}

Nous présentons ci-dessous les résultats des analyses univariée et multivariée qui ont permis de tester nos hypothèses et de vérifier la validité des échelles de mesure.

\section{Analyse univariée en France, en Chine et en Corée DU SUD}

\section{Des Échantillons majoritairement masculins}

L'échantillon français est constitué de 120 réponses aux questionnaires formulées par des praticiens et enseignants-chercheurs en management public. L'échantillon chinois est composé de 67 professeurs et professeurs adjoints en management public dans les quatre universités de la province de Jiangxi : Université de Nanchang, Université Jiujiang, École de gouvernement du Jiangxi et Université des Finances du Jiangxi. L'échantillon coréen, est

\begin{tabular}{|l|l|l|}
\hline \multicolumn{2}{|c|}{ Paradigme de Churchill (1979) pour de meilleures mesures } \\
\hline \multirow{2}{*}{ Principales phases } & Procédures & Techniques \\
\hline $\begin{array}{l}\text { 1. Définition du champ } \\
\text { conceptuel }\end{array}$ & 1. Spécifier le domaine du construit & Revue de littérature \\
\hline \multirow{2}{*}{ 2. Phase exploratoire } & 2. Génération d'un échantillon d'items & Revue de littérature + Enquête qualitative exploratoire \\
\cline { 2 - 3 } & 3. Première collecte des données & \\
\cline { 2 - 3 } & 4. Première purification de l'échelle de mesure & Analyse factorielle + Coefficient alpha de Chronbach \\
\hline \multirow{2}{*}{$\begin{array}{l}\text { 3. Phase confirmatoire validation } \\
\text { d. Deuxième collecte des données }\end{array}$} & Analyse factorielle Coefficient alpha de Chronbach \\
\cline { 2 - 3 } & $\begin{array}{l}\text { 6. Estimation de la fiabilité et purification de } \\
\text { l'échelle de mesure }\end{array}$ & Rho de Joreskög, Rho de validité convergente \\
\cline { 2 - 3 } & 7. Estimation de la validité & \\
\hline
\end{tabular}

\begin{tabular}{|l|c|c|c|c|c|c|}
\hline \multicolumn{7}{|c|}{$\begin{array}{c}\text { Age et ancienneté } \\
\text { Q12.2. Age (en années) }\end{array}$} \\
\hline \\
\hline
\end{tabular}

\begin{tabular}{|c|c|c|c|c|c|c|c|c|c|c|c|c|}
\hline \multicolumn{13}{|c|}{$\begin{array}{c}\text { TABLEAU } 3 \\
\text { Caractéristiques des répondants }(\%)\end{array}$} \\
\hline \multicolumn{4}{|c|}{$\begin{array}{l}\text { Q12.3. Niveau de diplôme } \\
\text { le plus élevé ( } \%)\end{array}$} & \multicolumn{3}{|c|}{$\begin{array}{l}\text { Q12.4. Avez-vous un } \\
\text { parent membre du } \\
\text { secteur public? ( } \%)\end{array}$} & \multicolumn{3}{|c|}{ Q12.1. Sexe } & \multicolumn{3}{|c|}{ Q12.8. Fonction } \\
\hline & $\mathbf{F}$ & C & Cor & $\mathbf{F}$ & C & Cor & $\mathbf{F}$ & C & Cor & $\mathbf{F}$ & C & Cor \\
\hline $\begin{array}{l}\text { PHD } \\
\text { doctorat }\end{array}$ & 70,50 & 24,60 & 5,00 & \multirow{4}{*}{54,08} & \multirow{4}{*}{29,10} & \multirow{4}{*}{42,5} & \multirow{2}{*}{$\begin{array}{l}\text { Homme } \\
\text { (70) }\end{array}$} & \multirow{2}{*}{$\begin{array}{l}\text { Homme } \\
(64,9)\end{array}$} & \multirow{2}{*}{$\begin{array}{l}\text { Homme } \\
(78,7)\end{array}$} & $\begin{array}{c}\text { Enseignants- } \\
\text { chercheurs } \\
(88,5)\end{array}$ & $\begin{array}{c}\text { Enseignants- } \\
\text { chercheurs } \\
(32,6)\end{array}$ & $\begin{array}{l}\text { Professeur } \\
\quad(57,7)\end{array}$ \\
\hline Master & 22,10 & 49,10 & 95,00 & & & & & & & $\begin{array}{c}\text { Enseignants } \\
\text { (10) }\end{array}$ & $\begin{array}{c}\text { Enseignants } \\
\text { (63) }\end{array}$ & $\begin{array}{l}\text { Chercheur } \\
\quad(38,2)\end{array}$ \\
\hline Licence & 7,40 & 21 & & & & & \multirow{2}{*}{$\begin{array}{l}\text { Femme } \\
\text { (30) }\end{array}$} & \multirow{2}{*}{$\begin{array}{l}\text { Femme } \\
(35,1)\end{array}$} & \multirow{2}{*}{$\begin{array}{c}\text { Femme } \\
(21,3)\end{array}$} & \multirow{2}{*}{ Autres $(1,5)$} & \multirow{2}{*}{ Autres $(4,4)$} & \multirow{2}{*}{$\begin{array}{c}\text { Autres } \\
(4,1)\end{array}$} \\
\hline Autres & 5,30 & 5,30 & & & & & & & & & & \\
\hline
\end{tabular}


composé de 127 praticiens et enseignants-chercheurs en management public. Si en France, les enseignants-chercheurs des universités sont fonctionnaires, en Chine ce sont des employés publics. En Corée du Sud, ils sont des enseignants-chercheurs, occupant des emplois publics et ayant pour la majorité d'entre eux, des formes de contrat de droit privé.

Les échantillons sont majoritairement composés d'hommes (70 \% en France; $65 \%$ en Chine; 78,7 \% en Corée du Sud). L'âge moyen est de 45 ans pour la France et la Corée du Sud, contre 36,66 ans pour la Chine. L'ancienneté moyenne est de 11 ans pour la Chine et la Corée du Sud et de 15 ans pour la France. Si $29 \%$ des répondants chinois ont au moins un de leurs parents ayant travaillé dans le secteur public, ils sont respectivement $54 \%$ et $42,5 \%$ en France et en Corée (voir les tableaux 2 et 3 ).

\section{Purification D'ÉCHElles}

Afin de purifier les échelles de mesure, nous effectuons sur les trois échantillons premièrement une analyse factorielle avec «rotation oblimin » et vérifions la stabilité de la structure factorielle pour les données françaises, coréennes et chinoises au moyen du critère de Kaiser. Nous ne conservons que les items dont la corrélation avec un axe est supérieure à 0,5 et éliminons les autres. Deuxièmement, nous examinons si chaque échelle de mesure contribue à rendre compte de façon fiable du concept étudié et purifions celles ayant un alpha de Cronbach inférieur à 0,5 (voir l'annexe 3 ).

\section{Echelles globalement fiables}

Nos échelles de mesure sont considérées comme globalement fiables à l'exception toutefois des échelles de devoir civiquesacrifice de soi et des valeurs de NPM (Q5.1, Q5.2, Q6.8 et Q6.7). Chaque échelle multiple a un alpha supérieur à 0,5 et la plupart ont un alpha supérieur à 0,70 . Deux items, Q1.6 et Q5.2, sont éliminés dans l'échantillon français en raison de leur faible contribution à l'échelle multiple respective. Dans le cas coréen, l'item Q8.1 de l'engagement calculé est exclu pour obtenir une fiabilité suffisante de l'échelle. Dans le cas français, les échelles de compassion et d'intérêt public constituent une seule dimension, alors qu' ils forment deux dimensions dans les cas chinois et coréen. Dans ces deux pays asiatiques, il semble y avoir une différence entre la compassion, attitude personnelle envers les autres en difficulté, et le comportement collectif dans la société qui appréhende la socialisation des membres.

\section{Analyse Comparative}

\section{Motivation affective : fort attachement à l'intérêt public et à la compassion}

Pour les échelles multiples considérées comme fiables, on retient comme variable mesurant le concept la moyenne des items de l'échelle. Nous comparons leurs valeurs entre les populations prises deux à deux en utilisant le test de Mann-Whitney. Le test de Kruskall-Wallis permet d'appréhender l'effet du facteur appartenance à une population sur la distribution d'une variable. Une différence est jugée significative dès lors que le niveau de signification du test unilatéral est inférieur à $5 \%$.

En analysant, les antécédents de la motivation affective, nous notons que la plupart des répondants français, chinois et coréens (environ plus de $80 \%$ d'entre eux) sont fortement ou très fortement touchés par l'intérêt pour le collectif et la compassion, (les réponses moyennes aux items correspondant de Q1.1 à Q1.6 sont assez élevées dans les trois pays).

L'attachement à l'intérêt général est plus élevé en Chine et en Corée qu'en France ${ }^{1}$. L'attachement des chinois à l'intérêt public (de Q2.1 à Q2.3) est le plus fort des trois échantillons². Dans les cultures collectivistes chinoises et coréennes, l'intérêt du groupe l'emporte sur les intérêts individuels.

\section{Motivation rationnelle : attachement général à la sécurité d'emploi et à la participation du public avec un sens collectif}

Nos résultats démontrent en fait un fort désir des répondants pour la sécurité d'emploi dans les trois pays (les valeurs moyennes des items de Q4.7 à Q4.9 liées à la sécurité d'emploi sont supérieures à 4), et ce encore plus en Chine et en Corée. Les chinois et les coréens valorisent encore plus la stabilité personnelle et la sécurité comme motif pour servir le secteur public car à la différence des français ils ne bénéficient pas du statut de fonctionnaire. Les chinois, coréens et français ont une assez forte attirance pour la chose publique (les moyennes des items $\mathrm{Q} 4.1$ à $\mathrm{Q} 4.3$ dépassent largement 3). Les résultats sur l'autonomie et le sentiment d'indépendance au travail (Q4.4 à Q4.6) montrent l'existence du sens collectif dans le travail partagé par les répondants des trois pays. Ce désir d'autonomie chinois est inférieur à celui français, lui-même inférieur à celui coréen ${ }^{3}$. Si la différence entre la France et la Chine peut s'expliquer par un plus grand individualisme chez les français, tel n'est pas le cas pour la différence entre coréens et français.

1. Utilisant le test de Kruskall-wallis, nous identifions avec un risque d'erreur inférieur à $1 \%$ une différence dans la distribution de la variable correspondant à l'intérêt général entre les trois populations.

2. En utilisant le test de Mann-Withney, nous concluons qu'il y a une différence entre les distributions de cette variable dans les deux populations (le niveau de signification du test est inférieur à $1 \%$ ).

3. En utilisant le test de Mann-Withney, nous concluons qu'il y a une différence entre les distributions de cette variable entre ces populations prises 2 à 2 (les niveaux de signification des tests sont inférieurs à $1 \%$ ).

4. http://www.clearlycultural.com/geert-hofstede-cultural-dimensions/uncertainty-avoidance-index.

5. A l'aide du test de Mann-Withney nous concluons qu'il y a une différence entre les distributions de ces variable dans les populations prises 2 à 2 (les niveaux de signification des tests sont inférieurs à $1 \%)$.

6. Les niveaux de signification des tests sont inférieurs à $1 \%$.

7. En utilisant le test de Mann-Withney nous concluons qu'il existe une différence entre les distributions de ces variables dans les deux populations, les niveaux des tests de signification sont inférieurs à $5 \%$.

8. En utilisant le test de Mann-Withney nous concluons qu'il existe une différence entre les distributions de ces variables dans les deux populations, les niveaux de signification des tests sont inférieurs à $5 \%$.

9. Le niveau de signification du test est inférieur à $1 \%$. 


\section{Motivation normative : attachement à la règle et respect de la tradition}

Dans les trois pays, les répondants ont un fort attachement à la règle : les réponses moyennes sont supérieures à 3 en France et à 4 en Chine et entre les deux pour la Corée. Ils le sont encore davantage en Corée et en Chine alors même que le score d'aversion à l'incertitude pour la France et la Corée du Sud respectivement égal à 86 et 85 , est plus élevé qu'en Chine où il est égal à $30^{4}$. Le respect de la tradition est, important dans les civilisations confucéennes qu'occidentales car les valeurs moyennes sont supérieures en Chine qu'en Corée et en Corée qu'en France ${ }^{5}$.

Les agents des trois échantillons sont assez fortement attachés aux valeurs du NPM (satisfaction des clients-usagers et bonne utilisation des impôts). Si l'attachement à la satisfaction des usagers est plus fort en France qu'en Corée, l'attachement au bon usage des impôts est plus faible en France ${ }^{6}$. Il n'y a pas de différences significatives si l'on compare chacun des deux pays avec la Chine.

\section{Engagement organisationnel : fort engagement affectif}

Si les répondants des trois pays ont un fort engagement affectif (les valeurs moyennes sont toutes proches de 4) il existe des divergences sur les engagements calculé et normatif. Les valeurs des items mesurant l'engagement normatif et calculé sont relativement faibles en France (scores inférieurs souvent à 3) alors qu'ils sont supérieurs à 3 en Chine ${ }^{7}$. Les Coréens ont un engagement affectif et calculé supérieur aux français ${ }^{8}$ et un engagement normatif inférieur aux Chinois ${ }^{9}$.

\section{Perception du contrôle sur le comportement :} forte auto-efficacité

La plupart des répondants ont un sens élevé de leur efficacité personnelle (les valeurs moyennes dans les trois pays sont largement au-dessus du point neutre 3). S'il n'y a pas de différence entre français et chinois, les coréens ont un sentiment d'efficacité personnelle plus faible ${ }^{10}$. Concernant la perception du soutien organisationnel, les français considèrent que leur contribution à l'organisation est mal prise en compte par leur employeur, alors que les chinois et les coréens semblent juger que celle-ci est mieux prise en compte ${ }^{11}$.

\section{L'ANALYSE MULTIVARIÉE}

Nous utilisons une analyse multivariée de type méthode des moindres carrés partiels (PLS) (Tenenhaus, 1998) qui peut se réaliser à partir de petits échantillons et permet d'intégrer des échelles formatives et réflexives. L'approche PLS permet de confirmer les qualités psychométriques des échelles et d'établir l'existence de relations entre les variables du modèle structurel. Nous établissons la significativité des relations du modèle théorique en vérifiant que le t de student, obtenu par bootstrap, et associé à chaque relation est supérieur à 2 .

\section{Analyse psychométrique des échelles}

Nous évaluons la fiabilité des échelles au moyen du $\rho$ de cohérence interne de Jöresckog (1971). L'échelle est considérée comme fiable dès lors que le $\rho$ est supérieur à 0,7 (Peterson, 1994). Le $\rho_{\mathrm{v}}$ de validité convergente permet d'évaluer la validité convergente des concepts réflexifs : l'échelle est dite valide dès lors que son $\rho_{\mathrm{v}}$ est supérieur à 0,5 . Le calcul du t de Student permet de déterminer si les relations de causalité entre motivation et engagement sont significatifs (la relation est significative dès lors que le t de Student est supérieur à 2) (voir l'annexe 3). Enfin, pour évaluer la validité discriminante, nous calculons le coefficient de détermination $\left(\mathrm{r}^{2}\right)$ de chaque variable réflexive et vérifions que le $r^{2}$ maximal de chaque concept réflexif est inférieur au $\rho$ de validité convergente $\rho$ (voir Tableau 4).

Tenant compte de tous les niveaux de signification, nous notons que :

- Les tests $t$ de Student montrent que les items contribuent significativement à chacune des échelles, ils sont tous significativement différents de 0 pour la France et pour la Chine. En revanche, pour la Corée du Sud, nous avons dû exclure l'item Q8.6 de l'échelle engagement normatif.

- Les mesures des construits endogènes ont une bonne valeur discriminante (chaque $r^{2}$ max est inférieur au $\rho$ de validité convergente pour les échantillons français, chinois et coréens).

\section{Analyse des relations causales}

Nos analyses montrent que (voir les figures 3, 4 et 5) :

- L'engagement affectif est correctement expliqué dans le cas français (le $r^{2}$ est de 0,33 ), chinois (le $r^{2}$ est de 0,38 ) et coréen (le $\mathrm{r}^{2}$ est de 0,314)

- L'engagement calculé est correctement expliqué dans le cas français (le $\mathrm{r}^{2}$ est 0,37 ), alors qu'il est bien expliqué dans le cas chinois (le $\mathrm{r}^{2}$ est de 0,54) et moyennement dans le cas coréen (le $\mathrm{r}^{2}$ est 0,17 )

\begin{tabular}{|l|c|c|c|c|c|c|}
\hline \multicolumn{7}{|c|}{ Tests de validité discriminante en France et en Chine } \\
\hline \multirow{2}{*}{ Concept } & \multicolumn{2}{c|}{ France } & \multicolumn{2}{c|}{ China } & \multicolumn{3}{c|}{ Corée } \\
\cline { 2 - 8 } & $\mathbf{r}^{2}$ Maximum & $\begin{array}{l}\rho \text { de validité } \\
\text { convergente }\end{array}$ & $\mathbf{r}^{2}$ Maximum & $\begin{array}{c}\rho \text { de validité } \\
\text { convergente }\end{array}$ & $\mathbf{r}^{2}$ Maximum & $\begin{array}{c}\rho \text { de validité } \\
\text { convergente }\end{array}$ \\
\hline Engagement affectif & 0,10 & 0,60 & 0,10 & 0,60 & 0,07 & 0,727 \\
\hline Engagement calculé & 0,01 & 0,55 & 0,18 & 0,64 & 0,08 & 0,666 \\
\hline Engagement normatif & 0,04 & 0,65 & 0,30 & 0,57 & 0,07 & 0,856 \\
\hline
\end{tabular}

10. Les niveaux de signification des tests sont inférieurs à $1 \%$.

11. La différence entre les deux populations est significative avec un risque de moins de $5 \%$. 


\section{FIGURE 3 \\ Les coefficients des relations significatives (France)}

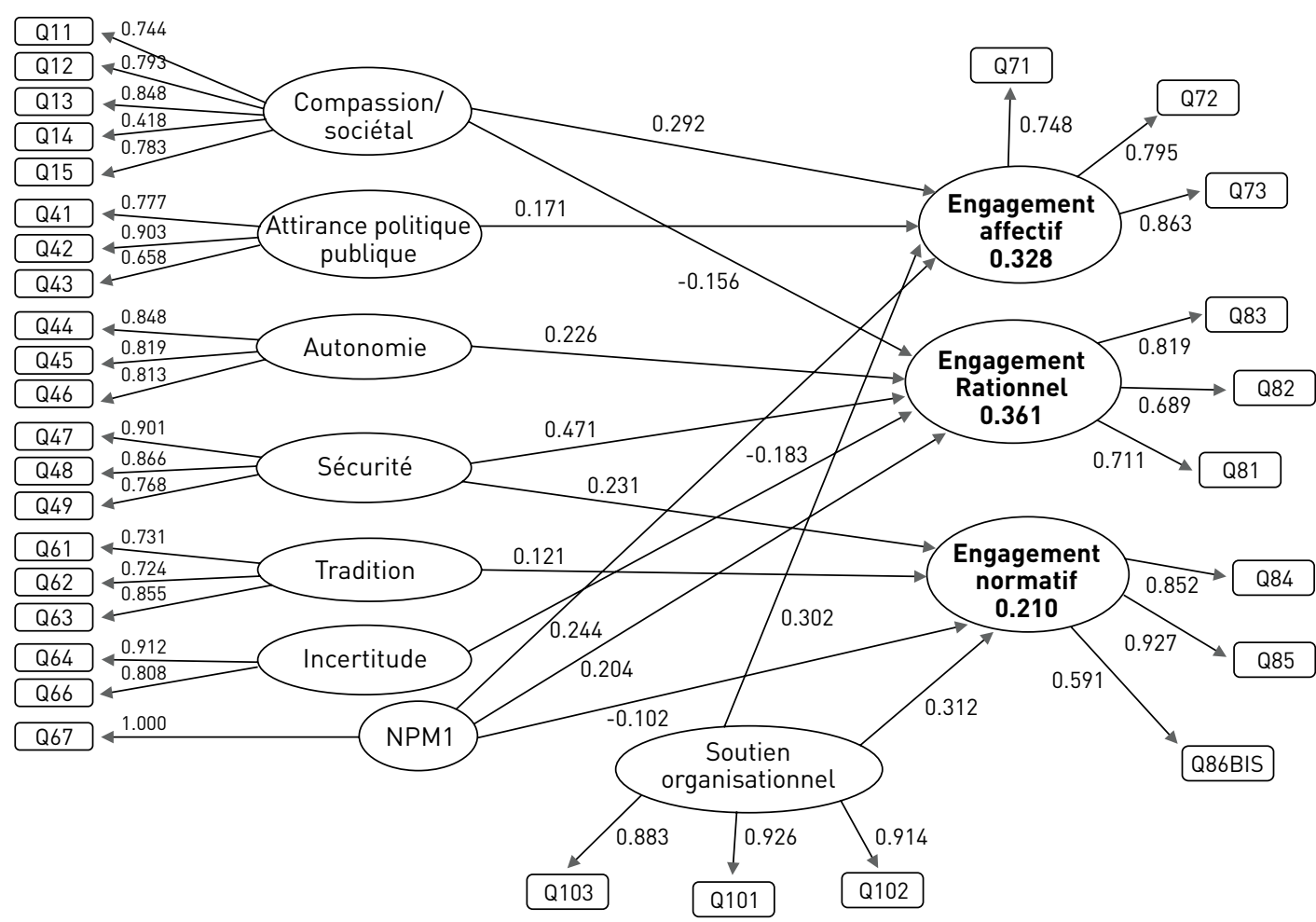

FIGURE 4

Les coefficients des relations significatives (Chine)

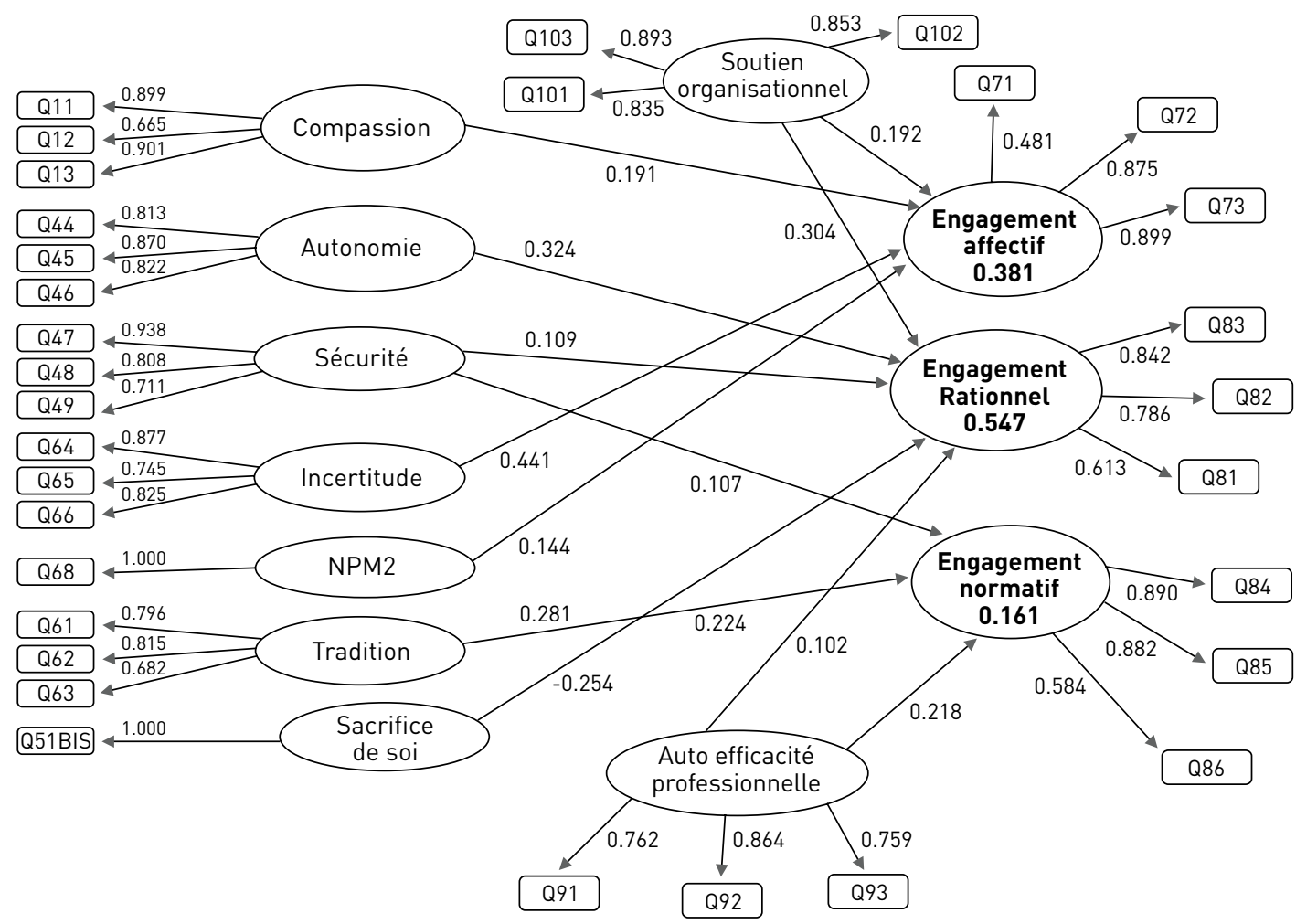


FIGURE 5

Les coefficients des relations significatives (Corée)

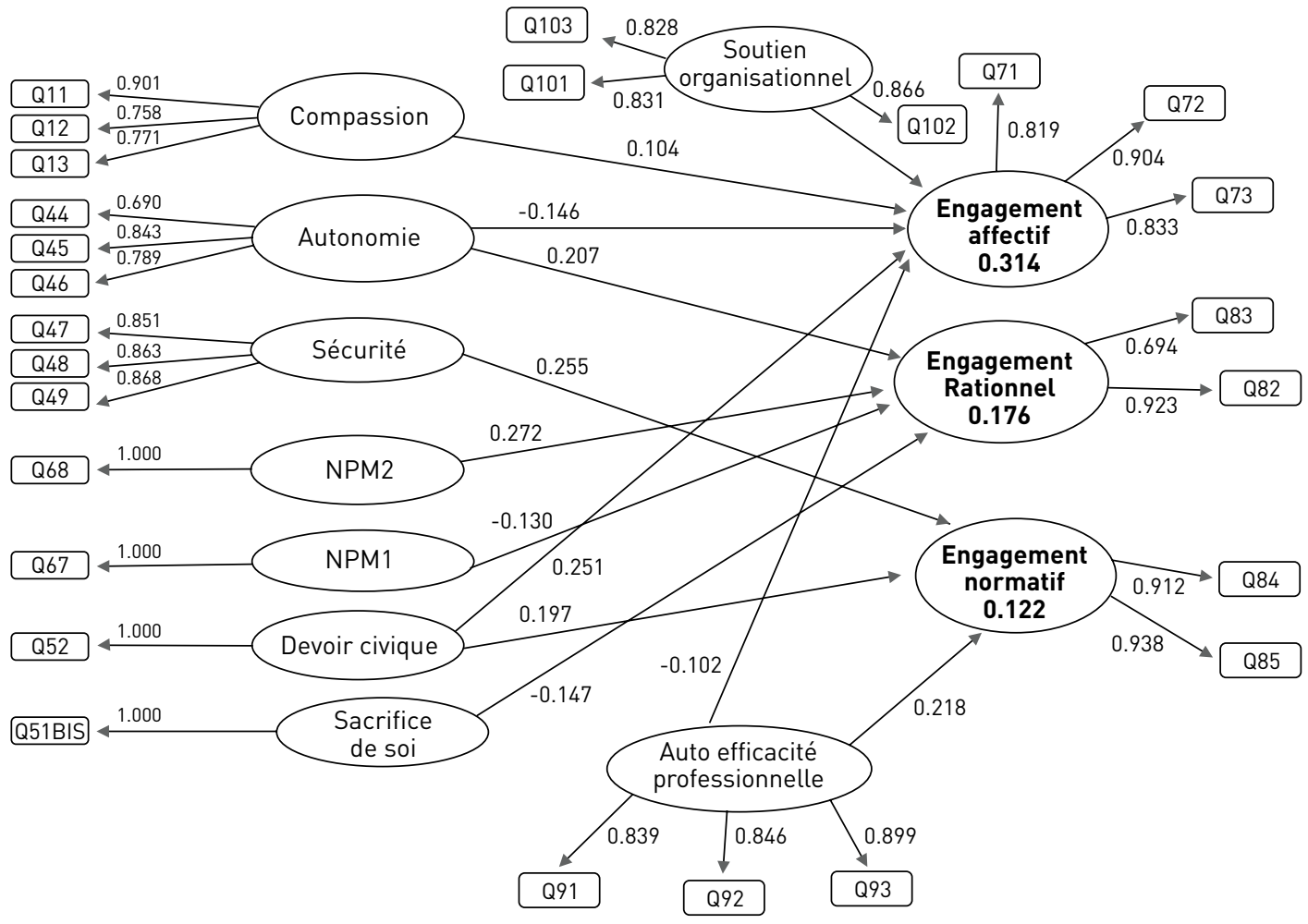

- L'engagement normatif est moyennement expliqué dans le cas français (le $\mathrm{r}^{2}$ est 0,21 ) et très moyennement expliqué dans le cas coréen (le $\mathrm{r}^{2}$ est 0,12 ), et dans le cas chinois (le $r^{2}$ est de 0,16$)$.

\begin{tabular}{|l|c|c|c|}
\hline \multicolumn{4}{|c|}{ TABLEAU 5 } \\
Variance expliquée de concepts endogènes (R2) \\
\hline \multirow{2}{*}{ Concept } & France & Chine & Corée \\
\cline { 2 - 4 } & $\mathbf{r}^{2}$ & $\mathbf{r}^{2}$ & $\mathbf{r}^{2}$ \\
\hline Engagement affectif & 0,33 & 0,38 & 0,31 \\
\hline Engagement calculé & 0,37 & 0,54 & 0,17 \\
\hline Engagement normatif & 0,21 & 0,16 & 0,12 \\
\hline
\end{tabular}

Nous présentons ci-dessous les relations de cause à effet entre les sources de motivation, la perception de contrôle sur le comportement et l'engagement pour le service public en France, en Chine et en Corée (les coefficients des relations entre les construits sont présentés sur les figures 3, 4 et 5). Les relations non significatives (au t de Student inférieur à 2) sont exclues des représentations. Nous remarquons que :
- Certains facteurs de MSP influencent non exclusivement le type d'engagement correspondant mais aussi d'autres types d'engagement. Certaines sources de motivation affective influent sur l'engagement affectif, mais aussi sur l'engagement calculé en France et en Chine.

- Les facteurs de motivation expliquent mieux les engagements affectif et calculé que normatif.

- En Chine, le sentiment d'auto-efficacité influe sur l'engagement calculé et normatif, alors qu'il n'a pas d'effet France et influe seulement sur l'engagement affectif en Corée.

- La perception du soutien organisationnel favorise l'engagement affectif dans les trois pays.

- La sécurité d'emploi influe sur l'engagement calculé en France et en Chine et sur l'engagement normatif en Corée.

\section{En France,}

- L'engagement affectif dépend d'un seul facteur de motivation affective (la compassion), rationnelle (l'attirance pour les politiques publiques) et normative (la satisfaction des usagers-clients du NPM). La perception du soutien organisationnel influence également l'engagement affectif.

12. Les niveaux de signification des tests sont inférieurs à $1 \%$.

13. La différence entre les deux populations est significative avec un risque de moins de $5 \%$.

14. Les tests $\mathrm{t}$ sont significatifs avec un risque d'erreur de moins de $1 \%$. 
- L'engagement calculé dépend principalement de deux facteurs de motivation rationnelle : l'autonomie et la sécurité d'emploi. Dans une certaine mesure, l'engagement calculé rejoint une vision individualiste de l'emploi où l'individu est motivé par la sécurité d'emploi et le sentiment d'autonomie dans son travail. Certains facteurs de motivation affective ou normative (la compassion ou l'évitement de l'incertitude) influent négativement sur l'engagement calculé. Il est d'autant plus fort, que le désir de faire un bon usage de l'impôt est élevé.

- L'engagement normatif dépend de deux facteurs normatifs (la tradition et le bon usage de l'impôt). Il dépend aussi de la perception du soutien organisationnel et du désir de sécurité d'emploi facteur de motivation rationnelle.

- Seule la perception du soutien organisationnel influe sur les trois formes d'engagement.

\section{En Chine,}

- L'engagement affectif dépend d'un seul facteur de motivation affective (la compassion) et de deux facteurs de motivation normative (l'évitement de l'incertitude et la satisfaction des besoins des usagers-clients). La perception du soutien organisationnel exerce une influence sur cet engagement.

- L'engagement calculé dépend principalement de facteurs de motivation rationnelle (l'autonomie et la sécurité d'emploi) mais aussi de la perception du soutien organisationnel et du sentiment d'auto-efficacité. Il est négativement lié à l'idée de sacrifice de l'intérêt individuel sur l'autel de l'intérêt général. Dans une certaine mesure, l'engagement calculé rejoint une vision individualiste de poursuite de l'intérêt personnel, notamment par la sécurité d'emploi et la reconnaissance du travail accompli individuellement.

- L'engagement normatif dépend d'un seul facteur de motivation normative (la tradition), mais aussi du sentiment d'auto-efficacité ${ }^{12}$ et d'un seul facteur rationnel (la sécurité d'emploi).

- Lauto-efficacité influe sur les engagements calculé et normatif, tandis que la perception du soutien organisationnel influe sur les engagements affectif et calculé.

\section{En Corée du Sud,}

- L'engagement affectif dépend de facteurs de motivation affective (la compassion) et normative (le devoir civique). Il est négativement lié au désir d'autonomie qui est une des dimensions rationnelles de la motivation ${ }^{13}$.

- L'engagement calculé dépend principalement d'un seul facteur de motivation rationnelle, l'autonomie. Il est négativement lié à la volonté de satisfaire les besoins des usagers-clients et au sacrifice de soi.

- L'engagement normatif dépend d'un seul facteur de motivation normative, le désir d'être un bon citoyen et d'un seul facteur de motivation rationnelle, la sécurité ${ }^{14}$. Le motif de sécurité d'emploi recherché par l'agent public aurait comme contrepartie d'un engagement moral auprès de son employeur.

- L'auto-efficacité et la perception du soutien organisationnel influent essentiellement sur l'engagement affectif.

\section{Conclusion}

Suivant la théorie du comportement planifié d'Ajzen (1971), nous construisons un modèle expliquant la relation entre les dimensions de la MSP et l'engagement des agents pour le service public. Nous incluons dans ce modèle les éléments dégagés par Perry, mais aussi des dimensions culturelles nationales et managériales comme la perception du soutien organisationnel ou l'adhésion à certains principes du NPM qui influent sur différentes formes d'engagement. L'étude empirique réalisée conjointement en Chine, France et Corée du Sud montre la constance de certaines relations entre des dimensions de la MSP et certains types d'engagement, mais aussi des différences provenant de spécificités culturelles conformément aux observations de Vandenabeele et Hondeghem (2005). Ainsi, si la quête de l'autonomie dans l'emploi influe sur l'engagement calculé dans les trois pays, l'attirance pour les politiques publiques n'influe significativement sur l'engagement normatif que pour la France. Le travail économétrique permet de valider nombre de mesures de motivation, mais aussi de dégager certains traits de motivation des agents publics propres ou tout au moins accentués dans certains pays. L'article retenant une approche internationale, complète, enrichit et élargit de l'analyse MSP en introduisant d'autres facteurs explicatifs de cette dynamique motivationnelle pour le service public.

Sur le plan managérial, ces analyses permettent d'identifier des leviers d'action pour une nouvelle gestion des ressources humaines propre permettant de développer l'engagement des agents publics. Les politiques de formation et de recrutement sont à lier aux formes d'engagements souhaités. La réalisation de tests de personnalité lors des recrutements ou d'oraux de concours permettant la révélation de la personnalité des individus au travers de mise en situation professionnelle permettraient d'identifier les recrues ayant les MSP souhaitées. Recruter des gens ayant de fortes valeurs altruistes et ayant par exemple développé antérieurement des actions dans le monde associatif peut permettre de développer l'engagement affectif. Inclure des actions de bénévolat dans les formations en management public peut également renforcer celui-ci. La mise en œuvre d'un management valorisant la reconnaissance des efforts déployés par l'agent peut aussi accroître cet engagement affectif. A contrario choisir des agents publics ayant le goût de la règle et les former à l'usage de la règle permet de développer l'engagement calculé. Maintenir et développer la sécurité de l'emploi au travers d'une politique statutaire qui est l'expression d'une relation asymétrique entre l'employeur public et l'agent public permet de s'assurer d'un fort engagement normatif. Le développement de modes de management mettant l'accent sur la tradition pourrait dans deux pays comme la France et la Chine de s'assurer un plus grand engagement normatif. Le prolongement naturel de cette étude qui fait partie d'un programme international de recherche sera de conduire une étude comparative entre secteur public et privé sur les antécédents de la motivation et leurs relations à l'engagement organisationnelle. Nous pourrons ainsi inclure les analyses d'Emery et Giauque (2012) sur l'impact des pratiques de management (enrichissement du travail, et participation aux prises de décision) sur la MSP, pratiques qui expliqueraient la perception du soutien organisationnel dans notre modèle. 


\section{Bibliographie}

Ajzen, I., \& Fishbein, M. (1980). Understanding attitudes and predicting social behavior. Englewood Cliffs, NJ : Prentice-Hall

Ajzen, L. (1991). «The theory of Planned Behavior», Organizational Behavior and Human Decision Processe, pp.179-211.

BAlfour D.L.; Wechsler, B. (1996) Organizational commitment; antecedents and outcomes in public organizations, Public Productivity and Management Review 19(3) : 256-77.

Bandura A. (1977), «Self-efficacy : Toward a Unifying Theory of Behavioral Change», Psychological Review, Vol. 84, $\mathrm{N}^{\circ} 2$, pp.191-215.

Bandura A. (1985), Social Foundations of Thought and Action : A Social Cognitive Theory, Prentice Hall.

Bateman T. S.; Strasser S. (1984), «A Longitudinal Analysis of the Antecedents of Organizational Commitment", Academy of Management Journal, 21, pp.95-112.

Baird L.S.; Mainstone, L.E. (1976), «The relationship of Job Level and Tenure to Individual Characteristics in a State Agency", Academic Management Proceedings, August, pp.479-482.

Bellah R. N.; Madsen R. Sullivan W. M; Swidler A.; \& Tipton S. M. (1986), "Habits of the Hart, Individualism and Commitment in American Life”, Perennial Library, USA.

Brunetto Y.; Xerri M.; Shriberg A.; Farr-Wharton R.; Shacklock K.; Newman S.; Dienger J. (2013) The impact of workplace relationships on engagement, well-being, commitment and turnover for nurses in Australia and the USA. Journal of Advanced Nursing 69(12), pp.2786-2799.

Churchill (1979), «A paradigm for Developing Better Measures of Marketing Construct», Journal of Marketing Research, Vol. XVI, February, pp.67-73.

Commeiras N. Fournier Ch. (2001), "Critical Evaluation of Porter et al.'s Organizational Commitment Questionnaire: Engagements for Researchers", The Journal of Personal Selling \& Sales Management, Summer 2001; 21; 3; pp.239-245.

Desmarais C.; Edey Gamassou C. (2014), «Tous motivés par le service public? Les liens entre position hiérarchique et motivation de service public», RISA, $2014:$ 1, vol. 80, pp.133-152.

Deter, J.R.; Schoeder, R.G.; Mauriel, J.J. (2000). «A framework for linking culture and improvement initiatives in organizations», The Academy of Management review, vol. 25, $\mathrm{N}^{\circ} 4$, pp.850-863.

Downs, A. (1967), "Inside bureaucracy”, Boston : Little, Brown.

Dunleavy P., Hood CH. (1994), From Old Public Administration to New Public Management, Public money and management, July-September, 9-16.

Dunn P., Shome A. (2009), «Cultural crossvergence and social desirability bias : Ethical evaluations by Chinese and Canadian business students", Journal of Business Ethics 85 (4), pp.527-543

Edey Gamassou C. (2014), «What drives personnel out of public organizations?», Public Organization Review, April 2014, pp.383-398.
Eisenberger R., Huntington R., Huntington S., Sowa, D. (1986), «Perceived organizational support», Journal of Applied Psychology, 71, pp.500-507.

Emery Y.; Giauque D. (2012) «Motivations et valeurs des agents publics à l'épreuve des réformes", Presses université Laval.

Fishbein, M.A.; Ajzen I. (1975). Belief, attitude, intention and behavior : an introduction to theory and research, Reading, MA, Addison Wesley.

Fons Trompenaars (1994), L'entreprise multiculturelle. Maxima

Frederickson H. G.; Hart D. K. (1985), «The public service and the patriotism of benevolence», Public Administration Review, Vol. 45 (5), pp.547-553.

Goncalo, J.A.; StaW, B.M. (2006), Individualism-collectivism and group creativity. Organizational Behavior and Human Decision Processes, 100, pp.96-109.

Harding, T.S.; Mayhew, M.M.; Finelli, C.J.; Carpenter, D.D. (2007, Sept). «The theory of planned behavior as a model of academic dishonesty in humanities and engineering undergraduate», Ethics and Behavior, vol. 17(3), pp.255-279.

Hofstede (1983), «National culture in four dimensions, a Researchbased Theory of Cultural Differences among Nations", International Studies of Management \& Organization, vol.13, $\mathrm{N}^{\circ} 1 / 2$, pp.46-74.

Hofstede G. (1984), “Culture's Consequences : International Differences in Work-Related Value” (2nd ed.), Beverly Hills CA, Sage publications.

Hofstede G. (1997), Cultures and Organizations : Software of the Mind, McGraw-Hill.

Hofstede G.; Hofstede G.J. (2005), Cultures and Organizations : Software of the Mind. 2nd Edition, McGraw-Hill USA.

Hofstede G.; Bond (1988), «The Confucius Connection : From cultural roots to economic growth », Organizational Dynamics, Spring 1988, Vol. 16, Issue 4, pp.5-21.

Hood CH. (1991), A public management for all seasons?, Public Administration, vol. 69, pp.3-19.

House, R. J.; Hanges, P. J.; Javidan, M.; Dorfman, P. W.; Gupta, V. (2004). «Culture, Leadership and Organizations: The GLOBE Study of 62 Societies», Sage Publications, Inc.

Julian S., Gould-Williams P., Bottomley T.R. (2013), “Civic duty and employee outcomes : do high commitment human resource practices and work overload matter?, Public Administration, Vol. 92, $\mathrm{N}^{\circ}$ 4, 2014, pp.937-953.

KIM (2005), “Individual Level Factors and Organizational Performance in Government Organizations", Journal of Public Administration Research and Theory, 15(2), pp.245-261.

KIM S., Vandenabeele et al. (2012), «Investigation the Structure and Meaning of Public Service across Populations : Developing an International Instrument and Addressing Issues of Measurement Invariance», Journal of Public Administration Research and Theory, 23 (1), pp.79-102.

Knoke, D.; Wright-Isak, C. (1982). "Individual motives and organizational incentive systems". Research in the Sociology of Organizations, 1, pp.209-254.

Mazouz, B.; Rousseau, A.; Sponem, S. (2015). «Le gestionnaire public en question », Revue française de gestion, $\mathrm{N}^{\circ} 250 / 2015$, pp.89-104. 
Meyer J. P; Stanley D. J.; Herscovitch L.; Topolnytsky L. (2002), "Affective, Continuance and Normative Commitment to the Organization: A Meta-analysis of Antecedents, Correlates, and Consequences", Journal of Vocational Behavior, Vol. 61, pp.20-52.

Meyer J. P; Allen N. (1991), "A three-component conceptualization of organizational commitment ", Human Resource Management Review, Vol. 1, $\mathrm{N}^{\circ}$ 1, pp.61-89.

Miller M., Woehr D.; Hudspeth N. (2002), «The meaning and measurement of work ethic: Construction and Initial validation of Multidimensional Inventory", Journal of Vocational Behavior, vol. $60, \mathrm{~N}^{\circ} 3$, June 2002, pp.451-489.

Niskanen, WA. (1971), Bureaucratie et gouvernement représentatif, Chicago : Aldine-Atherton.

Osborne D.; Gaebler T. (1993) “Reinventing Government. How the Entrepreneurial Spirit Is Transforming the Public Sector": A Plume Book, USA.

Paillé P., Fournier P-S., Lamontagne S. (2011), « Relationships between commitments to the organization, the superior and the colleagues, and the intention to leave among truckers", International Journal of Organizational Analysis, Vol. 19, $\mathrm{N}^{\circ} 2$, pp.92-108.

Pandey S.K.; Stazy K E.C. (2008) “Antecedents and Correlates of Public Service Motivation" in J. Perry and A. Hondeghem (eds), Motivation in public management. The call of public service. pp.101-117. Oxford University Press.

Perry J.L. (1996) «Measuring Public Service Motivation : An assessment of Construct Reliability and Validity", Journal of Public Administration Research and Theory, Vol. 6, $\mathrm{N}^{\circ} 1$, pp.5-22.

Perry J. L.(1997), «Antecedents of Public Service Motivation», Journal of Public Administration Research and Theory, Vol. 7, $\mathrm{N}^{\circ} 2$, pp.181-197.

Perry J. L.; Wise R. (1990), «The Motivational Bases of Public Service", Public Administration Review, May-June 1990, pp.367-373.

Pollitt, C.; Bouckaert, G. (2011). “Public Management Reform : A Comparative Analysis-New Public Management, Governance, and the Neo-Weberian State: Oxford", Oxford University Press, $365 \mathrm{p}$.

Pratchett, L. (1996). Petty bureaucracy and woolly-minded liberalism? The changing ethos of local government officers. Public administration, 74(4), 639.

Rainey, Hal G.; Steinbauer, P. (1999). «Galloping Elephants : Developing Elements of a Theory of Effective Government Organizations», Journal of Public Administration Research and Theory, 9, pp.1-32.

Ritz A. (2009), "La motivation de service public et la performance organisationnelle au sein de l'administration fédérale suisse», Revue Internationale des Sciences Administratives, 2009/1, Vol. 75, pp.59-86.

Robertson C.J.; Hoffman J.J. (2000), «How Different Are We? An Investigation of Confucian Values in the United States", Journal of Management, Vol. XII, N 1, pp.34-47.
Rokeach, M. (1973). The Nature of Human Values. New York : Free Press.

Sahertian P.; Soetjipto B. E. (2011), "Improving employee's organizational commitment, self-efficacy, and organizational citizenship behavior through the implementation of taskoriented and relationship-oriented leadership behavior", The Business Review, Cambridge, 17(2), pp. 48-60.

Shanock, S.; Eisenberger, R. (2006). "When Supervisors feel supported : Relationships with subordinates' perceived supervisor support, perceived organizational support and performance”, Journal of Applied Psychology, 91, pp. 689-695.

Shergold (2004), «Regeneration : New Structures, New Leaders, New Traditions ", Australian Journal of Public Administration, 54 (2), pp.3-6.

Shrestha A.K.; Mishra A. K. (2015), "Interactive effects of public service motivation and organizational politic on Nepali civil service employees' organizational commitment", Business Perspective and Research, 3(1), pp. 21-35.

Tenehaus M. (1998), La régression PLS : Théorie et pratique, Editions Technip.

The Chinese Culture Connection (1987), «Chinese Values and the Search for Culture-Free Dimensions of Culture», Journal of Cross-Cultural Psychology, 18, pp.143-164.

Tremblay M.; Guay Ph.; Simard G. (2000), «L'engagement organisationnel et les comportements discrétionnaires : l'influence des pratiques de gestion des ressources humaines», Publications du CIRANO, 24, pp. 1-24.

Vallerand, R.J.; Thill, E.E. (1993), "Introduction à la psychologie de la motivation", Laval : Editions Etudes Vivantes

Vandenabeele W. (2007), "Towards a Theory of Public Service Motivation : An Institutional Approach", Public Management Review, 9 : pp.545-556.

Vandenabeele W. (2009), “The Mediating Effect of Job Satisfaction and Organizational Commitment on Self-Reported Performance: More Robust Evidence of the PSM-Performance Relationship", International Review of Administrative Sciences, 75 : 11-34.

VANdenabeele W.; Hondeghem A. (2005), «Valeurs et motivation dans le service public», Revue française d'administration publique, 2005/3, N 115 , pp. 463-479.

VANDENABeEle W.; Van de WALle (2008), «International differences in public service motivation: Comparing regions across the world», dans, J.L. Perry et A. Houdeghem, Motivation in public management Oxford University Press, pp. 223-244.

Wright, B.E.; Christensen R; Isett K.R (2013), «Motivated to Adapt? The Role of Public Service Motivation as Employees Face Organizational Change", Public Administration Review, September-October 2013, vol.73, pp.738-747.

Wu, M. (2006), «Hofstede's Cultural Dimensions 30 Years Later : A Study of Taiwan and the United States", International Communication Studies, XV; 1, pp.33-42.

Xiaonua, L. (2008), “An empirical Study on Public Service Motivation and the Performance of Government Employee in China", Canadian Social Science, 4.2 : 18-28. 


\begin{tabular}{|c|c|c|c|c|c|c|}
\hline \multicolumn{7}{|c|}{$\begin{array}{c}\text { ANNEXE } 1 \\
\text { Questionnaire }\end{array}$} \\
\hline \multicolumn{7}{|c|}{ Dimensions affectives } \\
\hline & \multicolumn{6}{|l|}{ Compassion } \\
\hline & $\begin{array}{l}\text { On vous demande d'indiquer dans quelle mesure sur une échelle d'intensité de } 1 \text { à } 5 \text { (de pas du tout } \\
\text {... à ... extrêmement) }\end{array}$ & $\begin{array}{l}\text { Pas du tout } \\
\text { d'accord }\end{array}$ & Peu & $\begin{array}{l}\text { Dans une cer- } \\
\text { taine mesure }\end{array}$ & Fortement & Extrêmement \\
\hline Q1.1 & Vous êtes affecté par la situation des individus en situation difficile & 1 & 2 & 3 & 4 & 5 \\
\hline Q1.2 & Vous souhaiteriez aider les personnes en situation difficile & 1 & 2 & 3 & 4 & 5 \\
\hline \multirow[t]{2}{*}{ Q1.3 } & Vous vous souciez des difficultés rencontrées par des inconnus & 1 & 2 & 3 & 4 & 5 \\
\hline & \multicolumn{6}{|l|}{ Attitude face aux problèmes sociaux } \\
\hline Q1.4 & Vous aimez participer à des activités de bienfaisance & 1 & 2 & 3 & 4 & 5 \\
\hline Q1.5 & Vous-vous sentez concerner par les problèmes rencontrés par la société & 1 & 2 & 3 & 4 & 5 \\
\hline \multirow[t]{3}{*}{ Q1.6 } & Vous aimez les activités de groupe & 1 & 2 & 3 & 4 & 5 \\
\hline & \multicolumn{6}{|l|}{ Intérêt public } \\
\hline & $\begin{array}{l}\text { On vous demande d'indiquer par une échelle de } 1 \text { à } 5 \text { dans quelle mesure vous êtes d'accord avec les } \\
\text { propositions suivantes }\end{array}$ & $\begin{array}{l}\text { Pas du tout } \\
\text { d'accord }\end{array}$ & $\begin{array}{l}\text { Plutôt pas } \\
\text { d'accord }\end{array}$ & $\begin{array}{l}\text { Ni en accord ni } \\
\text { en désaccord }\end{array}$ & $\begin{array}{c}\text { Plutôt } \\
\text { d'accord }\end{array}$ & $\begin{array}{l}\text { Tout à fait } \\
\text { d'accord }\end{array}$ \\
\hline Q1.7 & Pour satisfaire mon intérêt personnel il est important d'améliorer le bien public & 1 & 2 & 3 & 4 & 5 \\
\hline Q1.8 & Mon intérêt particulier correspond toujours à l'intérêt général. & 1 & 2 & 3 & 4 & 5 \\
\hline Q1.9 & Améliorer le bien-être collectif est plus important qu'accroître mon propre bien être. & 1 & 2 & 3 & 4 & 5 \\
\hline \multicolumn{7}{|c|}{$\begin{array}{lll} & \text { Dimensions rationnelles }\end{array}$} \\
\hline & \multicolumn{6}{|l|}{ Attirance pour la politique publique } \\
\hline & $\begin{array}{l}\text { On vous demande d'indiquer par une échelle de } 1 \text { à } 5 \text { dans quelle mesure vous êtes d'accord avec les } \\
\text { propositions suivantes (de pas du tout d'accord.... à... tout à fait d'accord) }\end{array}$ & $\begin{array}{l}\text { Pas du tout } \\
\text { d'accord }\end{array}$ & $\begin{array}{l}\text { Plutôt pas } \\
\text { d'accord }\end{array}$ & $\begin{array}{l}\text { Ni en accord ni } \\
\text { en désaccord }\end{array}$ & $\begin{array}{l}\text { Plutôt } \\
\text { d'accord }\end{array}$ & $\begin{array}{l}\text { Tout à fait } \\
\text { d'accord }\end{array}$ \\
\hline Q4.1 & Contribuer aux programmes et politiques publics m'aide à me réaliser & 1 & 2 & 3 & 4 & 5 \\
\hline Q4.2 & Il est important pour moi de contribuer aux réformes qui améliorent le bien-être général & 1 & 2 & 3 & 4 & 5 \\
\hline \multirow[t]{2}{*}{ Q4.3 } & $\begin{array}{l}\text { J'ai besoin d'échanger avec les autres à propos des programmes et politiques publics pour savoir } \\
\text { comment produire service public plus performant }\end{array}$ & 1 & 2 & 3 & 4 & 5 \\
\hline & \multicolumn{6}{|l|}{ Autonomie } \\
\hline Q4.4 & J'ai besoin de tout faire moi-même & 1 & 2 & 3 & 4 & 5 \\
\hline Q4.5 & Je n'ai besoin d'aucune aide pour réussir mes projets & 1 & 2 & 3 & 4 & 5 \\
\hline \multirow[t]{2}{*}{ Q4.6 } & Les individus réussiraient mieux s'ils ne comptaient que sur eux-mêmes & 1 & 2 & 3 & 4 & 5 \\
\hline & \multicolumn{6}{|l|}{ Sécurité de l"emploi } \\
\hline Q4.7 & J'ai avant tout besoin de la sécurité dans mon travail & 1 & 2 & 3 & 4 & 5 \\
\hline Q4.8 & J'ai besoin d'être protégé dans l'exercice de mon activité professionnelle & 1 & 2 & 3 & 4 & 5 \\
\hline Q4.9 & J'ai besoin d'avoir l'assurance d'une carrière programmée & 1 & 2 & 3 & 4 & 5 \\
\hline
\end{tabular}




\begin{tabular}{|c|c|c|c|c|c|c|}
\hline \multicolumn{7}{|c|}{$\begin{array}{c}\text { ANNEXE } 1 \\
\text { Questionnaire }\end{array}$} \\
\hline \multicolumn{7}{|c|}{ Dimensions normatives } \\
\hline & \multicolumn{6}{|l|}{ Devoir civique et sacrifice de soi (dimension affective et normative) } \\
\hline & & $\begin{array}{l}\text { Pas du tout } \\
\text { d'accord }\end{array}$ & $\begin{array}{l}\text { Plutôt pas } \\
\text { d'accord }\end{array}$ & $\begin{array}{l}\text { Ni en accord ni } \\
\text { en désaccord }\end{array}$ & $\begin{array}{l}\text { Plutôt } \\
\text { d'accord }\end{array}$ & $\begin{array}{l}\text { Tout à fait } \\
\text { d'accord }\end{array}$ \\
\hline Q5.1 & Dans tous les cas, je ne sacrifierai jamais mon intérêt personnel au profit du bien être des autres & 1 & 2 & 3 & 4 & 5 \\
\hline \multirow[t]{3}{*}{ Q5.2 } & Pour être un meilleur citoyen, on se doit de contribuer au service public & 1 & 2 & 3 & 4 & 5 \\
\hline & \multicolumn{6}{|l|}{ Tradition/valeurs confucéennes } \\
\hline & $\begin{array}{l}\text { P/ On vous demande d'indiquer par une échelle de } 1 \text { à } 5 \text { dans quelle mesure vous êtes d'accord avec } \\
\text { les propositions suivantes (de pas du tout d'accord.... à... tout à fait d'accord) }\end{array}$ & $\begin{array}{l}\text { Pas du tout } \\
\text { d'accord }\end{array}$ & $\begin{array}{l}\text { Plutôt pas } \\
\text { d'accord }\end{array}$ & $\begin{array}{l}\text { Ni en accord ni } \\
\text { en désaccord }\end{array}$ & $\begin{array}{c}\text { Plutôt } \\
\text { d'accord }\end{array}$ & $\begin{array}{l}\text { Tout à fait } \\
\text { d'accord }\end{array}$ \\
\hline Q6.1 & Je me dois de respecter les traditions pour m'améliorer & 1 & 2 & 3 & 4 & 5 \\
\hline Q6.2 & Je dois écouter les ainés pour m'améliorer & 1 & 2 & 3 & 4 & 5 \\
\hline \multirow[t]{2}{*}{ Q6.3 } & Je dois adopter un comportement correspondant à ma position dans la société & 1 & 2 & 3 & 4 & 5 \\
\hline & \multicolumn{6}{|l|}{ Aversion pour l'incertitude et valeurs du NPM } \\
\hline 06.4 & $\begin{array}{l}\text { Il est important de définir en détail les exigences et instructions de travail afin que les } \\
\text { collaborateurs sachent ce qu'ils sont censés faire }\end{array}$ & 1 & 2 & 3 & 4 & 5 \\
\hline Q6.5 & Les cadres s'attendent à ce que leurs collaborateurs appliquent à la lettre instructions et procédures & 1 & 2 & 3 & 4 & 5 \\
\hline Q6.6 & Les modes opératoires normalisés sont utiles aux collaborateurs dans le cadre de leur travail & 1 & 2 & 3 & 4 & 5 \\
\hline Q6.7 & Le travail que je fais doit satisfaire le contribuable & 1 & 2 & 3 & 4 & 5 \\
\hline Q6.8 & Répondre aux besoins de la population est plus important que de respecter un ensemble de règles & 1 & 2 & 3 & 4 & 5 \\
\hline \multicolumn{7}{|c|}{ Engagement organisationnel pour le service public } \\
\hline & \multicolumn{6}{|l|}{ Engagement affectif } \\
\hline & $\begin{array}{l}\text { On vous demande d'indiquer dans quelle mesure sur une échelle d'intensité de } 1 \text { à } 5 \text { (de pas du tout } \\
\text {... à ... extrêmement) }\end{array}$ & Pas du tout & Peu & $\begin{array}{l}\text { Dans une cer- } \\
\text { taine mesure }\end{array}$ & Fortement & $\begin{array}{l}\text { Extrême- } \\
\text { ment }\end{array}$ \\
\hline Q7.1 & Vous êtes très heureux (se) de continuer votre carrière dans le secteur public & 1 & 2 & 3 & 4 & 5 \\
\hline Q7.2 & Vous vous sentez concerné par la performance de l'organisation publique à laquelle vous appartenez & 1 & 2 & 3 & 4 & 5 \\
\hline \multirow[t]{3}{*}{ Q7.3 } & Je me soucie de ce qui se passe dans le service public & 1 & 2 & 3 & 4 & 5 \\
\hline & \multicolumn{6}{|l|}{ Engagement calculé } \\
\hline & $\begin{array}{l}\text { On vous demande d'indiquer par une échelle de } 1 \text { à } 5 \text { dans quelle mesure vous êtes d'accord avec les } \\
\text { propositions suivantes (de pas du tout d'accord.... à... tout à fait d'accord) }\end{array}$ & $\begin{array}{l}\text { Pas du tout } \\
\text { d'accord }\end{array}$ & $\begin{array}{l}\text { Plutôt pas } \\
\text { d'accord }\end{array}$ & $\begin{array}{l}\text { Ni en accord ni } \\
\text { en désaccord }\end{array}$ & $\begin{array}{l}\text { Plutôt } \\
\text { d'accord }\end{array}$ & $\begin{array}{l}\text { Tout à fait } \\
\text { d'accord }\end{array}$ \\
\hline Q8.1 & J'ai trop investi ma carrière dans le secteur public, pour le quitter maintenant & 1 & 2 & 3 & 4 & 5 \\
\hline Q8.2 & Pour le moment travailler dans le secteur public est plus un choix rationnel, qu'un vrai désir & 1 & 2 & 3 & 4 & 5 \\
\hline Q8.3 & $\begin{array}{l}\text { Une des raisons principales pour laquelle je continue à travailler dans mon organisation est le } \\
\text { bénéfice du statut avantageux de fonctionnaire }\end{array}$ & 1 & 2 & 3 & 4 & 5 \\
\hline
\end{tabular}




\begin{tabular}{|c|c|c|c|c|c|c|}
\hline \multicolumn{7}{|c|}{$\begin{array}{c}\text { ANNEXE } 1 \\
\text { Questionnaire }\end{array}$} \\
\hline & \multicolumn{6}{|l|}{ Engagement normatif } \\
\hline Q8.4 & Je pense que je me dois de rester dans l'organisation publique dans laquelle j'évolue & 1 & 2 & 3 & 4 & 5 \\
\hline Q8.5 & Je pense que ce serait mal que de quitter le service public & 1 & 2 & 3 & 4 & 5 \\
\hline Q8.6 & Je ne dois rien à l'organisation publique à laquelle j'appartiens & 1 & 2 & 3 & 4 & 5 \\
\hline \multicolumn{7}{|c|}{ Perception du contrôle sur le comportement } \\
\hline & \multicolumn{6}{|l|}{ Auto-efficacité } \\
\hline & $\begin{array}{l}\text { On vous demande d'indiquer par une échelle de } 1 \text { à } 5 \text { dans quelle mesure vous êtes d'accord avec les } \\
\text { propositions suivantes (de pas du tout d'accord.... à... tout à fait d'accord) }\end{array}$ & $\begin{array}{l}\text { Pas du tout } \\
\text { d'accord }\end{array}$ & $\begin{array}{l}\text { Plutôt pas } \\
\text { d'accord }\end{array}$ & $\begin{array}{c}\mathrm{Ni} \text { en accord ni } \\
\text { en désaccord }\end{array}$ & $\begin{array}{c}\text { Plutôt } \\
\text { d'accord }\end{array}$ & $\begin{array}{l}\text { Tout à fait } \\
\text { d'accord }\end{array}$ \\
\hline Q9.1 & Je suis capable de faire ce que l'on attend de moi & 1 & 2 & 3 & 4 & 5 \\
\hline Q9.2 & J'ai le sentiment d'être efficace & 1 & 2 & 3 & 4 & 5 \\
\hline \multirow[t]{3}{*}{ Q9.3 } & Je suis très souvent satisfait de la qualité du travail que j’ai effectué & 1 & 2 & 3 & 4 & 5 \\
\hline & \multicolumn{6}{|l|}{ Perception du soutien organisationnel } \\
\hline & $\begin{array}{l}\text { On vous demande d'indiquer dans quelle mesure sur une échelle d'intensité de } 1 \text { à } 5 \\
\text { (de pas du tout à extrêmement) }\end{array}$ & Pas du tout & Peu & $\begin{array}{l}\text { Dans une cer- } \\
\text { taine mesure }\end{array}$ & Fortement & $\begin{array}{l}\text { Extrême- } \\
\text { ment }\end{array}$ \\
\hline Q10.1 & Votre organisation valorise bien votre contribution à sa réussite & 1 & 2 & 3 & 4 & 5 \\
\hline Q10.2 & Votre organisation est capable d'apprécier ce que vous faîtes et comment vous faîtes votre travail & 1 & 2 & 3 & 4 & 5 \\
\hline Q10.3 & Votre organisation vous écoute et prend en considération vos suggestions professionnelles & 1 & 2 & 3 & 4 & 5 \\
\hline \multicolumn{7}{|c|}{$\begin{array}{ll}2 & \text { Informations sociodémographiques } \\
\end{array}$} \\
\hline Q12.1 & \multicolumn{6}{|l|}{ Je suis un(e) homme/femme } \\
\hline Q12.2 & \multicolumn{6}{|l|}{ Mon âge au 1er janvier 2015} \\
\hline Q12.3 & \multicolumn{6}{|l|}{ Mon niveau de diplôme le plus élevé } \\
\hline Q12.4 & \multicolumn{6}{|l|}{ L'un de mes parents travaille lou a déjà travaillél dans une organisation oui/non } \\
\hline Q12.5 & \multicolumn{6}{|l|}{ Je travaille dans le secteur public/privé/autre secteur } \\
\hline Q12.6 & \multicolumn{6}{|l|}{ Depuis combien de temps travaillez-vous dans cette organisation (en années) } \\
\hline Q12.7 & \multicolumn{6}{|l|}{ Nombre de personnes dans l'organisation dans laquelle j'évolue : Moins de 10/10 à 100/Plus de 100} \\
\hline Q12.8 & \multicolumn{6}{|l|}{ Ma fonction dans l'organisation } \\
\hline Q12.9 & \multicolumn{6}{|l|}{ Commentaires supplémentaires } \\
\hline
\end{tabular}




\begin{tabular}{|c|c|c|c|c|c|c|}
\hline \multicolumn{7}{|c|}{$\begin{array}{c}\text { ANNEXE } 2 \\
\text { Moyenne des items et alpha de Cronbach }\end{array}$} \\
\hline \multirow[b]{2}{*}{ Items } & \multicolumn{3}{|c|}{ Moyenne } & \multicolumn{3}{|c|}{ Alpha de Cronbach } \\
\hline & France & Chine & Corée & France & Chine & Corée du Sud \\
\hline Q1.1 & 3,55 & 4,28 & 4,29 & \multirow{5}{*}{$\begin{array}{c}\text { Compassion et attitude } \\
\text { sociétale } \\
0,811\end{array}$} & \multirow{3}{*}{$\begin{array}{c}\text { Compassion } \\
0,785\end{array}$} & \multirow{3}{*}{$\begin{array}{c}\text { Compassion } \\
0,737\end{array}$} \\
\hline Q1.2 & 3,38 & 4,29 & 4,11 & & & \\
\hline Q1.3 & 3,11 & 4,09 & 3,55 & & & \\
\hline Q1.4 & 3,17 & 3,84 & 3,39 & & \multirow{3}{*}{$\begin{array}{l}\text { Attitude sociétale } \\
0,579\end{array}$} & \multirow{3}{*}{$\begin{array}{l}\text { Attitude sociétale } \\
\qquad 0,554\end{array}$} \\
\hline Q1.5 & 4,06 & 4,46 & 4,00 & & & \\
\hline Q1.6 & 3,17 & 4,37 & 3,12 & $\begin{array}{l}\text { Exclue de l'échelle après calcul } \\
\text { de l'alpha de cronbach }\end{array}$ & & \\
\hline Q2.1 & 2,72 & 4,02 & 3,33 & \multirow{3}{*}{$\begin{array}{l}\text { Intérêt public } \\
\quad 0,607\end{array}$} & \multirow{3}{*}{$\begin{array}{l}\text { Intérêt public } \\
0,796\end{array}$} & \multirow{3}{*}{$\begin{array}{l}\text { Intérêt public } \\
0,662\end{array}$} \\
\hline Q2.2 & 2,42 & 3,81 & 3,18 & & & \\
\hline Q2.3 & 3,67 & 4,16 & 3,60 & & & \\
\hline Q4.1 & 3,51 & 3,95 & 3,92 & \multirow{3}{*}{$\begin{array}{c}\text { Attirance pour la chose } \\
\text { publique } \\
0,796\end{array}$} & \multirow{3}{*}{$\begin{array}{c}\text { Attirance pour la chose } \\
\text { publique } \\
0,741\end{array}$} & \multirow{3}{*}{$\begin{array}{c}\text { Attirance pour la chose } \\
\text { publique } \\
0,814\end{array}$} \\
\hline Q4.2 & 3,86 & 4,04 & 3,99 & & & \\
\hline Q4.3 & 3,90 & 4,00 & 3,84 & & & \\
\hline Q4.4 & 2,77 & 3,02 & 3,31 & \multirow{3}{*}{$\begin{array}{l}\text { Autonomie } \\
0,773\end{array}$} & \multirow{3}{*}{$\begin{array}{l}\text { Autonomie } \\
0,784\end{array}$} & \multirow{3}{*}{$\begin{array}{l}\text { Autonomie } \\
0,655\end{array}$} \\
\hline Q4.5 & 2,01 & 2,51 & 2,12 & & & \\
\hline Q4.6 & 2,06 & 2,91 & 2,21 & & & \\
\hline Q4.7 & 3,35 & 4,33 & 4,05 & \multirow{3}{*}{$\begin{array}{l}\text { Sécurité de l'emploi } \\
\qquad 0,823\end{array}$} & \multirow{3}{*}{$\begin{array}{l}\text { Sécurité de l'emploi } \\
\qquad 0,759\end{array}$} & \multirow{3}{*}{$\begin{array}{l}\text { Sécurité de l'emploi } \\
\qquad 0,825\end{array}$} \\
\hline Q4.8 & 3,58 & 4,19 & 4,00 & & & \\
\hline Q4.9 & 3,62 & 4,19 & 4,05 & & & \\
\hline Q6.1 & 2,64 & 3,30 & 2,88 & \multirow{3}{*}{$\begin{array}{l}\text { Tradition } \\
0,709\end{array}$} & \multirow{3}{*}{$\begin{array}{l}\text { Tradition } \\
0,645\end{array}$} & \\
\hline Q6.2 & 2,97 & 2,77 & 3,23 & & & $\begin{array}{l}\text { Tradition } \\
0.748\end{array}$ \\
\hline Q6.3 & 2,61 & 4,02 & 3,10 & & & \\
\hline 06.4 & 3,58 & 4,32 & 3,74 & & & \\
\hline Q6.5 & 3,16 & 4,28 & 3,81 & $\begin{array}{c}\text { Aversion pour l'incertitude } \\
0.723\end{array}$ & $\begin{array}{c}\text { Aversion pour l'incertitude } \\
0.748\end{array}$ & $\begin{array}{l}\text { Aversion pour l'incertitude } \\
0.673\end{array}$ \\
\hline Q6.6 & 3,67 & 4,14 & 3,91 & & & \\
\hline Q5.1 & 3,99 & 2,58 & 2,47 & Echelles simples dites du & Echelles simples dites du & Echelles simples dites du \\
\hline 05.2 & 3,85 & 4,30 & 3,90 & devoir civique et du sacrifice de & devoir civique et du sacrifice de & devoir civique et du sacrifice de \\
\hline Q6.7 & 4,04 & 4,11 & 3,84 & $\begin{array}{l}\text { soi, échelle multiple du NPM } \\
\text { correspondant à ces items non }\end{array}$ & $\begin{array}{l}\text { soi, échelle multiple du NPM } \\
\text { correspondant à ces items non }\end{array}$ & $\begin{array}{l}\text { soi, échelle multiple du NPM } \\
\text { correspondant à ces items non }\end{array}$ \\
\hline Q6.8 & 3,68 & 3,39 & 2,85 & fiables car alpha inférieur à 0,5 & fiables car alpha inférieur à 0,5 & fiables car alpha inférieur à 0,5 \\
\hline Q7.1 & 3,61 & 3,86 & 3,90 & & & \\
\hline Q7.2 & 4,00 & 4,21 & 4,03 & $\begin{array}{l}\text { Engagement affectif } \\
0,682\end{array}$ & $\begin{array}{l}\text { Engagement affectif } \\
0,648\end{array}$ & $\begin{array}{c}\text { Engagement affectif } \\
0,801\end{array}$ \\
\hline Q7.3 & 4,12 & 4,09 & 4,11 & & & \\
\hline Q8.1 & 3,21 & 3,19 & 2,85 & & & \\
\hline Q8.2 & 2,60 & 3,25 & 3,03 & $\begin{array}{l}\text { Engagement calculé } \\
0,62\end{array}$ & $\begin{array}{l}\text { Engagement calculé } \\
0,609\end{array}$ & Engagement calculé \\
\hline Q8.3 & 2,81 & 3,19 & 3,14 & & & 0,54 \\
\hline Q8.4 & 2,68 & 3,26 & 3,03 & & & \\
\hline Q8.5 & 2,50 & 3,05 & 2,65 & $\begin{array}{l}\text { Engagement normatif } \\
0,719\end{array}$ & $\begin{array}{l}\text { Engagement normatif } \\
0,733\end{array}$ & $\begin{array}{l}\text { Engagement normatif } \\
0,59\end{array}$ \\
\hline Q8.6 & 3,58 & 3,46 & 2,84 & & & \\
\hline Q9.1 & 4,12 & 4,05 & 4,00 & & & \\
\hline Q9.2 & 4,20 & 3,98 & 3,74 & $\begin{array}{l}\text { Auto-efficacité } \\
0.725\end{array}$ & $\begin{array}{l}\text { Auto-efficacité } \\
0,645\end{array}$ & $\begin{array}{c}\text { Auto-efficacité } \\
0.824\end{array}$ \\
\hline Q9.3 & 3,83 & 4,02 & 3,62 & & & \\
\hline Q10.1 & 2,43 & 3,23 & 3,18 & Perception du soutien & Perception du soutien & Perception du soutien \\
\hline Q10.2 & 2,60 & 3,33 & 3,18 & organisationnel & organisationnel & organisationnel \\
\hline Q10.3 & 2,66 & 3,02 & 3,03 & 0,893 & 0,748 & \\
\hline
\end{tabular}




\begin{tabular}{|c|c|c|c|c|c|c|c|c|c|c|c|c|c|}
\hline \multirow[b]{3}{*}{ Concept } & \multirow[b]{3}{*}{ Item } & \multicolumn{11}{|c|}{$\begin{array}{l}\text { ANNEXE } 3 \\
\text { Qualités psychométriques des échelles multiples inclues dans les modèles définitifs } \\
\text { (sont exclues les variables qui n'ont aucun effet significatif sur l'Engagement) }\end{array}$} & \\
\hline & & \multicolumn{4}{|c|}{ France } & \multicolumn{4}{|c|}{ Chine } & \multicolumn{4}{|c|}{ Corée du Sud } \\
\hline & & Coeff & Student's t & $\rho$ reliability & $\rho$ conv. val. & Coeff & Student's t & $\rho$ reliability & $\rho$ conv. val. & Coeff & Student's t & $\rho$ reliability & p conv. val. \\
\hline \multirow[t]{3}{*}{ Compassion } & Q1.1 & 0,77 & 20,10 & \multirow{5}{*}{0,85} & \multirow{5}{*}{0,55} & 0,90 & 40,11 & \multirow{3}{*}{0,87} & \multirow{3}{*}{0,69} & 0,901 & 20,53 & \multirow{3}{*}{0,852} & \multirow{3}{*}{0,756} \\
\hline & Q1.2 & 0,79 & 20,07 & & & 0,67 & 9,71 & & & 0,758 & 7,57 & & \\
\hline & Q1.3 & 0,85 & 32,02 & & & 0,90 & 47,24 & & & 0,771 & 6,02 & & \\
\hline \multirow[t]{3}{*}{ Attitude sociétale } & Q1.4 & 0,42 & 4,52 & & & \multirow{3}{*}{\multicolumn{4}{|c|}{ Variable non significative }} & \multirow{3}{*}{\multicolumn{4}{|c|}{ Variable non significative }} \\
\hline & Q1.5 & 0,78 & 19,11 & & & & & & & & & & \\
\hline & Q1.6 & \multicolumn{4}{|c|}{ Item exclu de l'échelle lépuration de l'échelle) } & & & & & & & & \\
\hline \multirow[t]{3}{*}{ Intérêt publique } & Q2.1 & \multirow{3}{*}{\multicolumn{4}{|c|}{ Variable non significative }} & \multirow{3}{*}{\multicolumn{4}{|c|}{ Variable non significative }} & \multirow{3}{*}{\multicolumn{4}{|c|}{ Variable non significative }} \\
\hline & Q2.2 & & & & & & & & & & & & \\
\hline & Q2.3 & & & & & & & & & & & & \\
\hline \multirow{3}{*}{$\begin{array}{l}\text { Attirance pour les } \\
\text { politiques publiques }\end{array}$} & Q4.1 & 0,78 & 15,72 & \multirow{3}{*}{0,88} & \multirow{3}{*}{0,72} & \multirow{3}{*}{\multicolumn{4}{|c|}{ Variable non significative }} & \multirow{3}{*}{\multicolumn{4}{|c|}{ Variable non significative }} \\
\hline & Q4.2 & 0,90 & 54,87 & & & & & & & & & & \\
\hline & Q4.3 & 0,86 & 35,45 & & & & & & & & & & \\
\hline \multirow[t]{3}{*}{ Autonomie } & Q4.4 & 0,85 & 23,20 & \multirow{3}{*}{0,87} & & 0,81 & 40,54 & & & 0,690 & 4,01 & & \\
\hline & Q4.5 & 0,82 & 17,24 & & 0,68 & 0,87 & 75,79 & 0,87 & 0,70 & 0,843 & 3,24 & 0,819 & 0,603 \\
\hline & Q4.6 & 0,81 & 17,56 & & & 0,82 & 30,07 & & & 0,789 & 3,79 & & \\
\hline Sécurité de l'emploi & Q4.7 & 0,90 & 91,69 & & & 0,94 & 24,74 & & & 0,852 & 10,57 & & \\
\hline & Q4.8 & 0,86 & 46,59 & 0,88 & 0,72 & 0,81 & 9,53 & 0,86 & 0,68 & 0,861 & 14,65 & 0,895 & 0,74 \\
\hline & Q4.9 & 0,77 & 17,93 & & & 0,71 & 7,62 & & & 0,868 & 10,81 & & \\
\hline $\begin{array}{l}\text { Devoir civique et } \\
\text { sacrifice de soi }\end{array}$ & $\begin{array}{c}\text { Q5.1 } \\
\text { (reverse) }\end{array}$ & Variak & les non signif & ficatives et éch & tle multiple & 1,00 & $\begin{array}{l}\text { Echelle } \\
\text { simple }\end{array}$ & Echelle m & ـltiple non & 1 & $\begin{array}{l}\text { Echelle } \\
\text { simple }\end{array}$ & Echelle m & ıltiple non \\
\hline & Q5.2 & & non fiable Var & iable non sign & icative & 1 & $\begin{array}{l}\text { Echelle } \\
\text { simple }\end{array}$ & & & & & fia & \\
\hline Tradition/valeurs & Q6.1 & 0,73 & 6,87 & & & 0,80 & 13,99 & & & & & & \\
\hline confucéennes & Q6.2 & 0,72 & 7,09 & 0,82 & 0,60 & 0,81 & 16,54 & 0,81 & 0,60 & & Variable & non significati & \\
\hline & Q6.3 & 0,86 & 13,33 & & & 0,68 & 8,19 & & & & & & \\
\hline Aversion pour & Q6.4 & 0,91 & 3,01 & 085 & & 0,88 & 61,59 & & & & & & \\
\hline l'incertitude & Q6.6 & 0,81 & 2,43 & 0,85 & 0,74 & 0,75 & 21,43 & 086 & 0.67 & & Variable & non sianificati & \\
\hline & Q6.5 & Item & $\begin{array}{l}\text { exclu car } \mathrm{t} d \mathrm{e} \\
\text { significative }\end{array}$ & $\begin{array}{l}\text { student infér } \\
\text { ment différen }\end{array}$ & $\begin{array}{l}\text { ur à } 2 \text { non } \\
\text { de } 0\end{array}$ & 0,82 & 33,73 & & & & & & \\
\hline
\end{tabular}

ualités psychométriques des échelles multiples inclues dans les modèles définitifs 


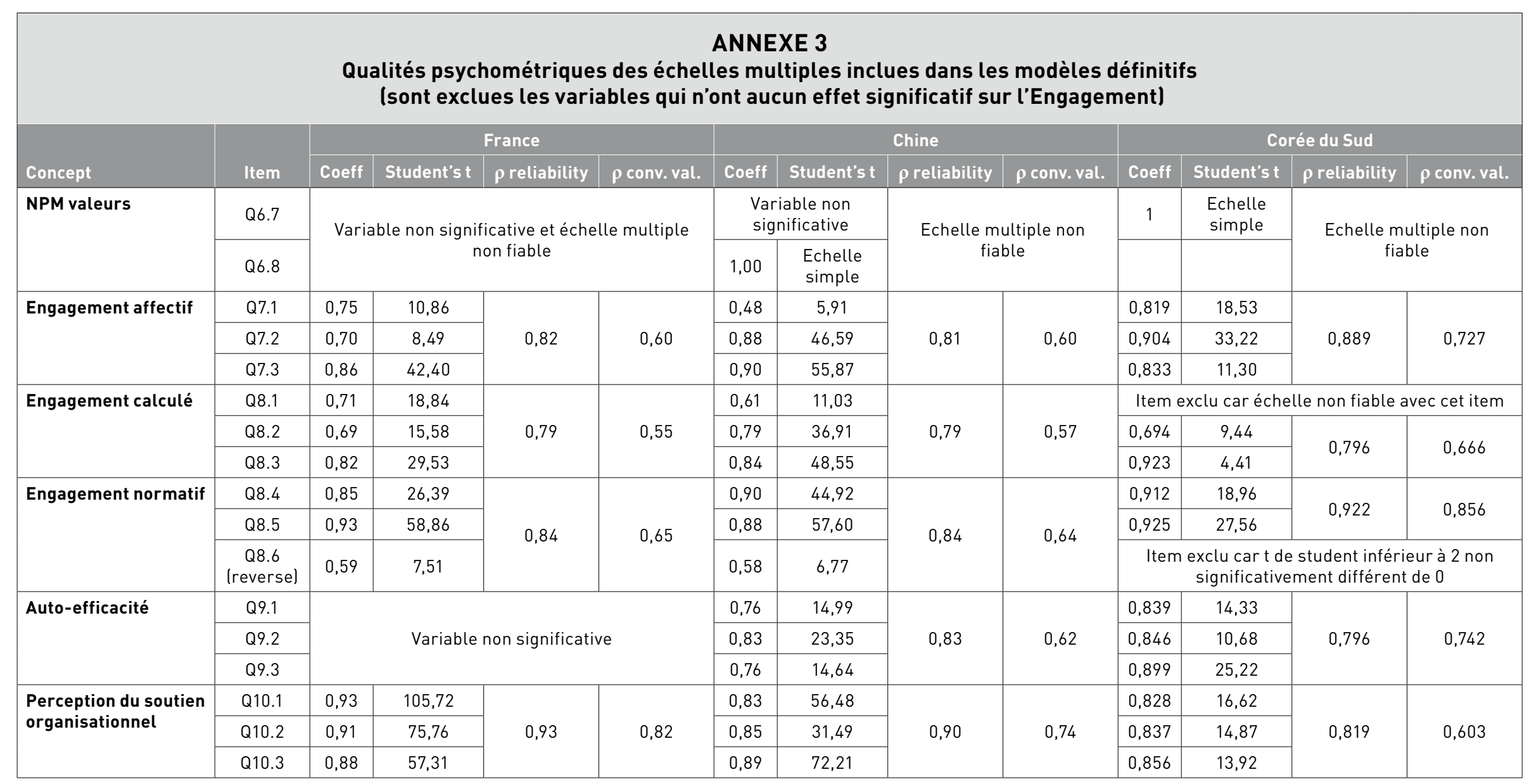

